Leandro Gonçalves

Propriedades Reflexivas das Cônicas

Dissertação apresentada como requisito parcial para obtenção do grau de Mestre pelo Programa de Pós-graduação em Matemática do Departamento de Matemática da PUC-Rio

Orientadora: Prof. Renata Martins da Rosa 
Leandro Gonçalves

\title{
Propriedades Reflexivas das Cônicas
}

Dissertação apresentada como requisito parcial para obtenção do grau de Mestre pelo Programa de Pós-graduação em Matemática do Departamento de Matemática do Centro Técnico Científico da PUC-Rio. Aprovada pela Comissão Examinadora abaixo assinada.

\author{
Prof. Renata Martins da Rosa \\ Orientadora \\ Departamento de Matemática - PUC-Rio
}

Prof. Humberto José Bortolossi

Instituto de Matemática - UFF

Prof. Sinésio Pesco

Departamento de Matemática - PUC-Rio

Prof. Débora Freire Mondaini

Departamento de Matemática - PUC-Rio

Prof. José Eugenio Leal

Coordenador Setorial do Centro Técnico Científico - PUC-Rio

Rio de Janeiro, 28 de Março de 2014 
Todos os direitos reservados. É proibida a reprodução total ou parcial do trabalho sem autorização da universidade, do autor e do orientador.

\section{Leandro Gonçalves}

Graduado em Bacharelado em Matemática pela Universidade Federal do Rio de Janeiro em 2006. Graduado em Licenciatura em Matemática pela Universidade Federal do Rio de Janeiro em 2007.

Trabalhou em colégios particulares e cursos preparatórios (Pré Vestibular e Pré Militar). Atualmente está lotado no Colégio Pedro II - Campus São Cristóvão II e possui uma matrícula na Rede Municipal de Ensino da cidade do Rio de Janeiro na escola municipal Gaspar Vianna.

Realizou cursos de aperfeiçoamento para professores do Ensino Médio junto ao Instituto Nacional de Matemática Pura e Aplicada (IMPA) nos anos de 2006 e 2007.

Ficha Catalográfica

Gonçalves, Leandro

Propriedades Reflexivas das Cônicas / Leandro Gonçalves; orientador: Renata Martins da Rosa. - Rio de Janeiro : PUCRio, Departamento de Matemática, 2014.

v., 52 f: il. ; $29,7 \mathrm{~cm}$

1. Dissertação (mestrado) - Pontifícia Universidade Católica do Rio de Janeiro, Departamento de Matemática.

Inclui referências bibliográficas.

1. Matemática - Dissertação. 2. Parábola 3. Elipse

4. Hipérbole 5. Propriedades Reflexivas das Cônicas I. Rosa, Renata Martins d. II. Pontifícia Universidade Católica do Rio de Janeiro. Departamento de Matemática. III. Propriedades Reflexivas das Cônicas.

CDD: 510 


\section{Agradecimentos}

A Deus por ter me dado luz, calma, paciência e sabedoria para concluir o curso.

A minha orientadora Renata Martins da Rosa pela paciência incomensurável, atenção e sabedoria que me acompanhou nesse período de elaboração e confecção do nosso trabalho final de conclusão de curso. Obrigado por tudo, professora Renata.

Ao pessoal do departamento de Matemática pela ajuda e paciência ao longo desses dois anos. Em particular à secretaria Creuza Nascimento e ao prof. Sinésio Pesco.

Em especial aos meus pais pelo apoio incondicional, carinho e apoio de sempre. Graças a eles, conquistei tudo o que pude almejar até agora. Obrigado por tudo pais: Angela de Souza Gonçalves e Eloy Mandarino Gonçalves.

Em especial à minha noiva, Aline Natália de Oliveira Gomes, meu grande amor da minha vida e por toda a eternidade, minha inspiração. Sem você, nada disso seria possível. Você é a razão da minha vida. Obrigado por tudo minha linda.

Em especial aos meus grandes e ilustríssimos amigos de mestrado. A ajuda de vocês foi proveitosa em todos os sentidos, tanto na parte emocional quanto na parte acadêmica. Com vocês pude compartilhar experiências e amizades que com certeza irão se perdurar ao longo das nossas vidas. Amigos, muito obrigado por tudo.

Aos meus amigos de trabalho, que me passaram muita força, sabedoria, conhecimento. Em especial, ao meu grande amigo e coordenador Daniel Martins, que sempre esteve ao meu lado, me orientando com suas sábias palavras ao longo desses dois anos. Obrigado por tudo meu irmão. 


\section{Resumo}

Gonçalves, Leandro; Rosa, Renata Martins d. Propriedades Reflexivas das Cônicas. Rio de Janeiro, 2014. 52p. Dissertação de Mestrado - Departamento de Matemática, Pontifícia Universidade Católica do Rio de Janeiro.

A ideia principal desta dissertação é apresentar as cônicas e demonstrar suas equações cartesianas bem como suas propriedades reflexivas. O trabalho está focado em abordar tais propriedades reflexivas com o auxílio do software GeoGebra.

\section{Palavras-chave}

Parábola; Elipse; Hipérbole; Propriedades Reflexivas. 


\section{Abstract}

Gonçalves, Leandro; Rosa, Renata Martins d. Reflective

Properties of Conics. Rio de Janeiro, 2014. 52p.

MSc. Dissertation - Departamento de Matemática, Pontifícia Universidade Católica do Rio de Janeiro.

The main idea of this dissertation is to present the conics and demonstrate their cartesian equations and their reflective properties. The work is focused on addressing such reflective properties with the aid of software GeoGebra.

\section{Keywords}

Parabola; Ellipse; Hyperbola; Reflective Properties. 


\section{Sumário}

1 Introdução $\quad 8$

2 Parábola $\quad 10$

3 Elipse $\quad 15$

4 Hipérbole $\quad 25$

5 Aplicações $\quad 38$

$6 \quad$ Projeto de Ensino $\quad 43$

7 Conclusão e trabalhos futuros $\quad 46$

$\begin{array}{ll}\text { Referências Bibliográficas } & 47\end{array}$

A Apêndice com Recíproca da Eq. da Elipse $\quad 48$

B Apêndice com Recíproca da Eq. da Hipérbole $\quad 51$ 


\section{1 \\ Introdução}

A ideia principal deste trabalho é apresentar e demonstrar as propriedades reflexivas das cônicas, a fim de entendermos o motivo pelo qual alguns objetos e aparelhos que refletem luz, som ou sinais de rádio e TV são construídos de forma que suas seções tenham formato de parábola, elipse ou hipérbole como, por exemplo, uma antena parabólica. Tal trabalho tem um caráter interdisciplinar e revela a grande importância de se estudar as propriedades reflexivas das cônicas.

Definimos cada cônica como lugar geométrico de pontos do plano e não usamos seção de cone. Nos capítulos 1, 2 e 3 deduzimos as equações cartesianas de parábola, elipse e hipérbole e demonstramos suas respectivas propriedades reflexivas. A demonstração que apresentamos é analítica e usa derivada. Essa abordagem proporcionou a possibilidade de explorar argumentos e ferramentas da disciplina do Profmat de cálculo a uma variável como, por exemplo, equação da reta tangente ao gráfico. Demonstrações geométricas e sem derivada, onde a reta tangente é definida pelo número de pontos de interseção, são mais comuns na literatura (Oliveira) e (Sato).

No capítulo 4, além da antena parabólica, apresentamos e justificamos várias aplicações das propriedades reflexivas. Como essas aplicações fazem parte do cotidiano, elas podem despertar o interesse dos alunos do ensino médio pelo estudo das cônicas, tendo uma relação direta com a Física no que diz respeito à Óptica Geométrica.

É muito importante também que o aluno, ao deixar o ensino médio, tenha a noção de cônica bem definida como lugar geométrico de pontos do plano que cumprem certas propriedades. Isso em geral não acontece ao longo do ensino médio, quando os professores apresentam as cônicas de forma apenas algébrica, e em alguns casos, não demonstrando de fato porque tais equações são daquele tipo. Um exemplo clássico é quando o professor do $1^{\circ}$ ano do ensino médio, ao introduzir o gráfico de uma função quadrática, afirma que a equação $y=a x^{2}+b x+c$ representa uma parábola, o que é verdade, porém existe a necessidade do professor comentar e demonstrar que tal equação representa uma parábola, isto é, que essa equação representa um lugar geométrico no plano.

Este trabalho consiste, em parte, de um projeto futuro a ser desenvolvido na escola em que trabalho, de forma a contribuir com a melhor compreensão 
e visualização de conceitos e elementos importantes quando tratamos sobre esse tópico no ensino médio. No projeto usaremos um software de geometria dinâmica (no nosso caso, o GeoGebra). Uma grande vantagem de se utilizar um programa como o Geogebra é o poder de movimentação de objetos proporcionando uma boa visualização sobre o problema que nós estamos tratando. O GeoGebra irá nos auxiliar nesse âmbito fazendo o uso de funções importantes como o cálculo de distâncias de ponto a ponto e de ponto à reta como forma de verificação das propriedades das cônicas. Com exceção das figuras do capítulo 4, todas as figuras desse trabalho foram feitas com o GeoGebra justamente para evitar o uso de figuras deformadas. O texto desse trabalho é encerrado com um esboço de parte desse projeto de ensino no capítulo 5 . 


\section{2}

\section{Parábola}

Uma parábola é o conjunto dos pontos do plano que equidistam de uma reta fixa, dita diretriz, e de um ponto fixo fora da reta, dito foco. A reta que passa pelo foco e é perpendicular à reta diretriz é dita eixo da parábola e o ponto médio entre o foco e o ponto de interseção da reta diretriz com o eixo é dito vértice da parábola.

\section{1}

\section{Equação Cartesiana}

Sejam $F$ um ponto e $r$ uma reta no plano tais que $F \notin r$. Vamos deduzir a equação cartesiana da parábola de foco $F$ e diretriz $r$. Para facilitar as contas, o eixo y será o eixo da parábola e o vértice da parábola será a origem. Com isso $F=(0, p)$ e a equação da diretriz é $y=-p$, onde $p$ é a distância do foco ao vértice.

Considere $P_{1}=(x, y)$ um ponto pertencente à parábola e $P_{2}=(x,-p)$ o pé da perpendicular baixada do ponto $P_{1}=(x, y)$ em relação a reta diretriz. Pela definição da parábola, sabemos que a distância entre $P_{1}$ e $F$, denotada por $\operatorname{dist}\left(P_{1}, F\right)$, é igual à distância entre $P_{1}$ e $P_{2}$, denotada por $\operatorname{dist}\left(P_{1}, P_{2}\right)$.

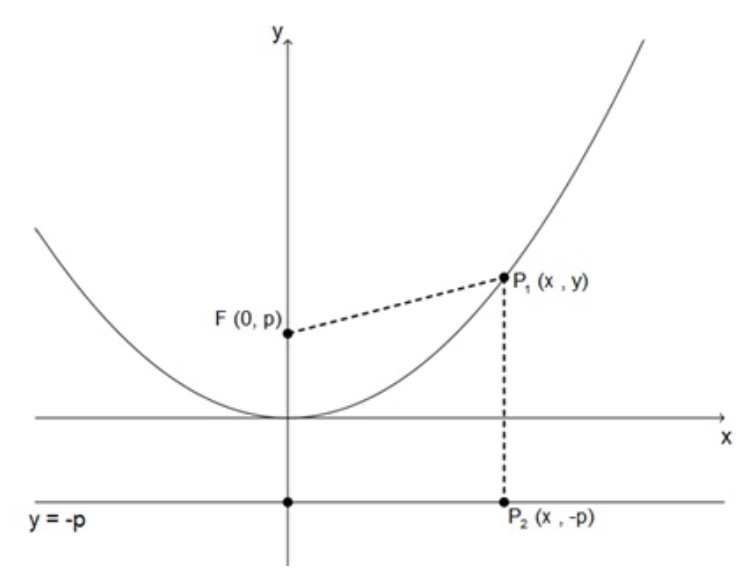

Figura 2.1: Parábola Definição

Efetuando os cálculos:

$\operatorname{dist}\left(P_{1}, F\right)=\operatorname{dist}\left(P_{1}, P_{2}\right)$,

$\Rightarrow \sqrt{(x-0)^{2}+(y-p)^{2}}=\sqrt{(x-x)^{2}+(y-(-p))^{2}}$.

Elevando ao quadrado ambos os lados da equação acima, temos:

$(x-0)^{2}+(y-p)^{2}=(x-x)^{2}+(y-(-p))^{2}$, 


$$
\begin{aligned}
& \Rightarrow x^{2}+y^{2}-2 y p+p^{2}=0^{2}+(y+p)^{2}, \\
& \Rightarrow x^{2}+y^{2}-2 y p+p^{2}=y^{2}+2 y p+p^{2}, \\
& \Rightarrow 4 y p=x^{2}
\end{aligned}
$$

Finalmente

$$
y=\frac{x^{2}}{4 p} .
$$

Reciprocamente, se as coordenadas de um ponto $P=(x, y)$ satisfazem a equação $y=\frac{x^{2}}{4 p}$ para algum $p>0$, então o ponto $P$ pertence à parábola de foco $(0, p)$ e diretriz de equação $y=-p$. Para provar essa recíproca basta seguir as contas acima no caminho inverso.

\section{2}

\section{Propriedade reflexiva}

\subsection{1}

\section{Reflexão}

Antes de falarmos sobre as propriedades reflexivas das cônicas, vamos definir uma reflexão de uma reta em relação a uma curva suave qualquer no ponto $P$. Uma reflexão de uma reta (ou segmento de reta ou ainda semirreta) num ponto $P$ qualquer da curva é uma outra reta na qual o ângulo de incidência em relação à reta tangente que passa por $P$ é igual ao ângulo de reflexão. Para entendermos melhor, vejamos a figura abaixo:

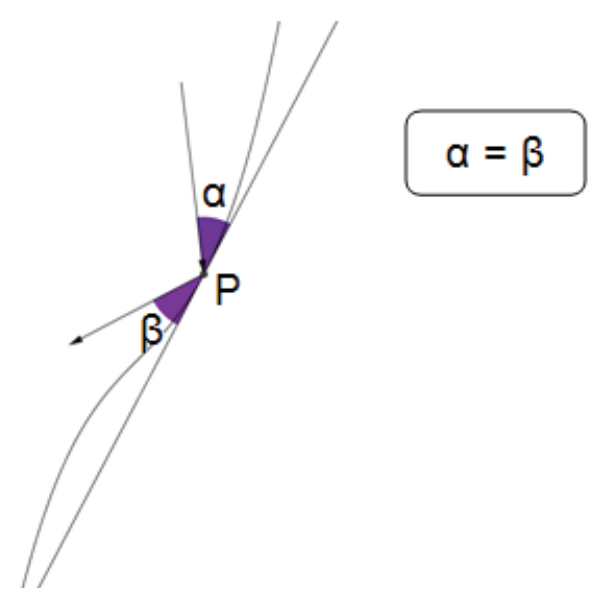

Figura 2.2: Reflexão 


\subsection{2}

\section{Propriedade reflexiva da parábola}

Vamos agora mostrar a propriedade reflexiva da parábola. Ela nos diz que se uma reta é paralela ao eixo da parábola, então sua reflexão passará pelo foco da parábola.

Primeiramente vamos encontrar a equação da reta tangente em um ponto da parábola. A parábola é o gráfico da função

$$
\begin{aligned}
f: \mathbb{R} & \longrightarrow \mathbb{R} \\
x & \longmapsto f(x)=\frac{x^{2}}{4 p} .
\end{aligned}
$$

Esta função é derivável em todos os pontos e sua derivada em $x_{0}$ é $f^{\prime}\left(x_{0}\right)=$ $\frac{2 x_{0}}{4 p}=\frac{x_{0}}{2 p}$. A equação da reta tangente ao gráfico da função $f$ no ponto $P=\left(x_{0}, y_{0}\right)$ é $y-y_{0}=\frac{x_{0}}{2 p}\left(x-x_{0}\right)$.

Seja $Q$ o ponto de interseção entre o eixo $y$ e a reta tangente em $P$, digamos $t$, como mostra a Figura 2.3. Escrevemos $Q=(0,-q)$.

Como $P=\left(x_{0}, y_{0}\right)$ pertence à parábola, temos $y_{0}=\frac{x_{0}^{2}}{4 p}$. E como $Q=(0,-q)$ pertence à reta $t$, temos

$$
\begin{aligned}
& -q-\frac{x_{0}^{2}}{4 p}=\frac{x_{0}}{2 p}\left(0-x_{0}\right) \\
& \Rightarrow q=\frac{x_{0}^{2}}{2 p}-\frac{x_{0}^{2}}{4 p} \\
& \Rightarrow q=\frac{x_{0}^{2}}{4 p} .
\end{aligned}
$$

Então, temos que:

$$
Q=\left(0,-\frac{x_{0}^{2}}{4 p}\right) \quad \text { e } P=\left(x_{0}, \frac{x_{0}^{2}}{4 p}\right) .
$$

Seja $\alpha$ o ângulo formado pela reta paralela ao eixo da parábola passando por $P$ e a reta $t$, e $\beta$ o ângulo formado pela reta que passa pelos pontos $F$ e $P$ e a reta $t$ tais que $\alpha$ e $\beta$ estejam contidos no mesmo semiplano determinado pela reta $t$ e que contém o foco $F$, como na figura abaixo.

Observe que o ângulo entre o eixo $y$ e a reta $t$ também é $\alpha$. Devemos mostrar que $\alpha=\beta$. Em particular, quando $P$ é o vértice da parábola, a reta passando por $F$ e $P$ e a reta passando por $P$ e paralela ao eixo da parábola são 


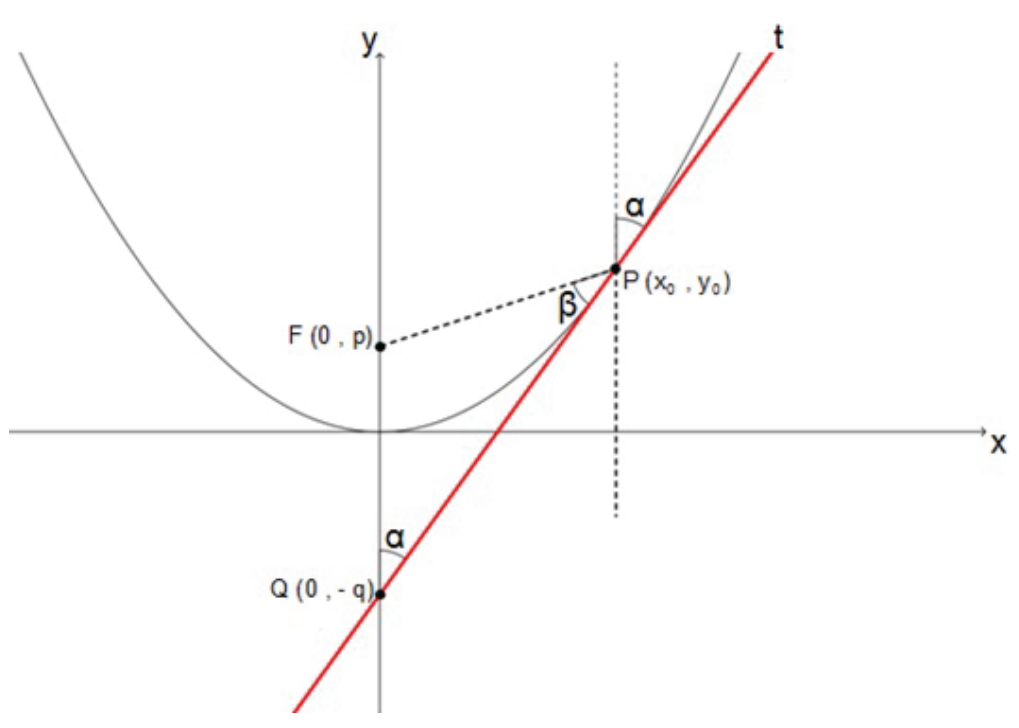

Figura 2.3: Propriedade Reflexiva Parábola

o próprio eixo $y$, portanto $\alpha$ e $\beta$ são iguais nesse caso. Se $P$ não é o vértice, basta mostrar que o triângulo $Q F P$ é isósceles de base $Q P$. Com efeito, observe que:

$F Q=\sqrt{(0-0)^{2}+(p-(-q))^{2}}$

$\Rightarrow F Q=\sqrt{0^{2}+(p+q)^{2}}$

$\Rightarrow F Q=\sqrt{0+(p+q)^{2}}$

$\Rightarrow F Q=\sqrt{(p+q)^{2}}$

$\Rightarrow F Q=|p+q|$.

Como $p>0$ e $q=\frac{x_{0}^{2}}{4 p}>0$, temos $p+q>0$ e $|p+q|=p+q$, portanto:

$$
F Q=p+q=p+\frac{x_{0}^{2}}{4 p} .
$$

Agora, observe que:

$$
\begin{aligned}
& F P=\sqrt{\left(0-x_{0}\right)^{2}+\left(p-y_{0}\right)^{2}} \\
& \Rightarrow F P=\sqrt{\left(-x_{0}\right)^{2}+\left(p-\frac{x_{0}^{2}}{4 p}\right)^{2}} \\
& \Rightarrow F P=\sqrt{x_{0}^{2}+p^{2}-2 p\left(\frac{x_{0}^{2}}{4 p}\right)+\left(\frac{x_{0}^{2}}{4 p}\right)^{2}} \\
& \Rightarrow F P=\sqrt{p^{2}+x_{0}^{2}-\frac{2 p x_{0}^{2}}{4 p}+\frac{x_{0}^{4}}{16 p^{2}}} \\
& \Rightarrow F P=\sqrt{p^{2}+\frac{4 p x_{0}^{2}-2 p x_{0}^{2}}{4 p}+\frac{x_{0}}{16 p^{2}}}
\end{aligned}
$$




$$
\begin{aligned}
& \Rightarrow F P=\sqrt{p^{2}+\frac{2 p x_{0}^{2}}{4 p}+\frac{x_{0}{ }^{4}}{16 p^{2}}} \\
& \Rightarrow F P=\sqrt{p^{2}+2 p \frac{x_{0}^{2}}{4 p}+\left(\frac{x_{0}^{2}}{4 p}\right)^{2}} \\
& \Rightarrow F P=\sqrt{\left(p+\frac{x_{0}^{2}}{4 p}\right)^{2}} \\
& \Rightarrow F P=\left|p+\frac{x_{0}^{2}}{4 p}\right| .
\end{aligned}
$$

Como $p>0$ e $\frac{x_{0}^{2}}{4 p} \geq 0$, então $p+\frac{x_{0}^{2}}{4 p}>0$ e $\left|p+\frac{x_{0}^{2}}{4 p}\right|=p+\frac{x_{0}^{2}}{4 p}$. Logo,

$$
F P=p+\frac{x_{0}^{2}}{4 p}
$$

Com isso, concluímos que $F P=F Q$, e segue-se daí que o triângulo $Q F P$ é isósceles de base $Q P$. Portanto,

$$
\alpha=\beta
$$




\section{Elipse}

Uma elipse é o conjunto dos pontos do plano cuja soma das distâncias a dois pontos fixos, ditos focos, é constante e essa constante é maior que a distância entre os focos.

\section{1}

\section{Equação cartesiana da elipse}

Sejam $F_{1}$ e $F_{2}$ dois pontos distintos ${ }^{1}$ no plano e $a>0$ tal que $\operatorname{dist}\left(F_{1}, F_{2}\right)<2 a$. Vamos deduzir a equação cartesiana da elipse com focos $F_{1}$ e $F_{2}$ e constante $2 a$. Para facilitar as contas, o eixo $x$ será a reta que passa pelos focos e o eixo $y$ a reta perpendicular que passa pelo ponto médio dos focos. Com isso, podemos escrever $F_{1}=(c, 0)$ e $F_{2}=(-c, 0)$, onde $2 c$ é a distância entre os focos. Lembramos que $2 a>2 c$, ou seja, $a>c$.

Considere $P=(x, y)$ um ponto pertencente à elipse. Pela definição, sabemos que

$$
\operatorname{dist}\left(P, F_{1}\right)+\operatorname{dist}\left(P, F_{2}\right)=2 a .
$$

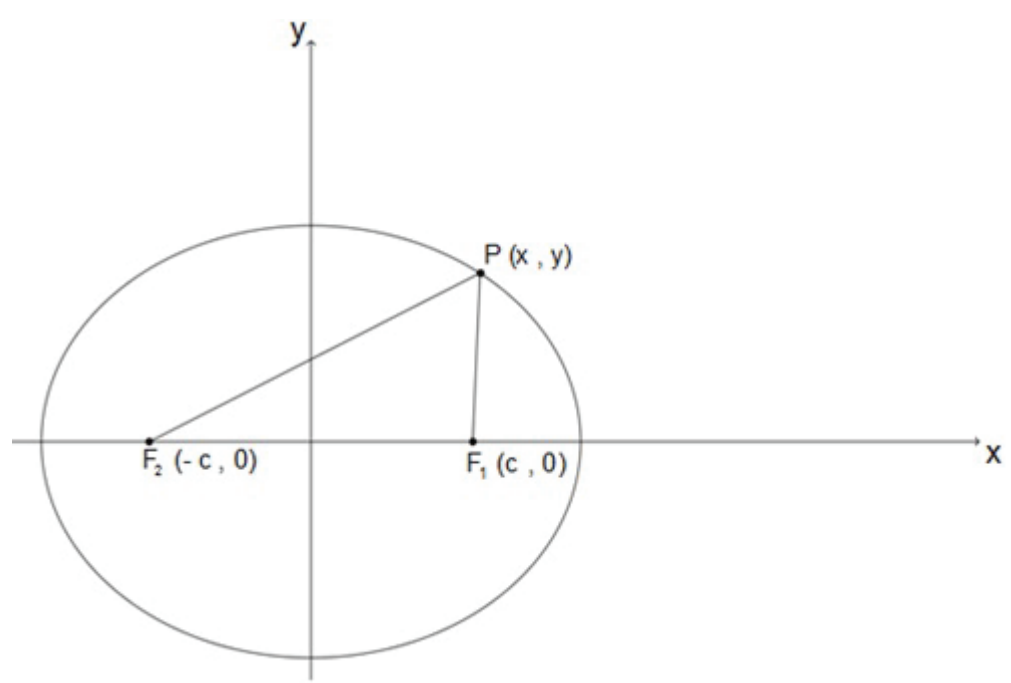

Figura 3.1: Elipse Definição

Efetuando os cálculos:

$$
\begin{aligned}
& \operatorname{dist}\left(P, F_{1}\right)+\operatorname{dist}\left(P, F_{2}\right)=2 a \\
& \Rightarrow \sqrt{(x-c)^{2}+y^{2}}+\sqrt{(x+c)^{2}+y^{2}}=2 a
\end{aligned}
$$

${ }^{1}$ Não trabalhamos com o caso $F_{1}=F_{2}$, ou seja, não trabalhamos com circunferência, pois a propriedade reflexiva da circunferência não é interessante. 


$$
\Rightarrow \quad \sqrt{(x-c)^{2}+y^{2}}=2 a-\sqrt{(x+c)^{2}+y^{2}} .
$$

Elevando ao quadrado ambos os lados da equação acima, temos:

$$
\begin{aligned}
& \left(\sqrt{(x-c)^{2}+y^{2}}\right)^{2}=\left(2 a-\sqrt{(x+c)^{2}+y^{2}}\right)^{2} \\
& \Rightarrow(x-c)^{2}+y^{2}=\left(2 a-\sqrt{(x+c)^{2}+y^{2}}\right)^{2} \\
& \Rightarrow x^{2}-2 x c+c^{2}+y^{2}=4 a^{2}-4 a \sqrt{(x+c)^{2}+y^{2}}+\left(\sqrt{(x+c)^{2}+y^{2}}\right)^{2} \\
& \Rightarrow x^{2}-2 x c+c^{2}+y^{2}=4 a^{2}-4 a \sqrt{(x+c)^{2}+y^{2}}+(x+c)^{2}+y^{2} \\
& \Rightarrow 4 a \sqrt{(x+c)^{2}+y^{2}}=4 a^{2}+x^{2}+2 x c+c^{2}-c^{2}+y^{2}-y^{2}-x^{2}+2 x c \\
& \Rightarrow 4 a \sqrt{(x+c)^{2}+y^{2}}=4 a^{2}+4 x c .
\end{aligned}
$$

Simplificando, temos:

$a \sqrt{(x+c)^{2}+y^{2}}=a^{2}+x c$.

Elevando ao quadrado ambos os lados da equação acima, temos:

$$
\begin{aligned}
& a^{2}\left(\sqrt{(x+c)^{2}+y^{2}}\right)^{2}=\left(a^{2}+x c\right)^{2} \\
& \Rightarrow a^{2}\left((x+c)^{2}+y^{2}\right)=\left(a^{2}+x c\right)^{2} \\
& \Rightarrow a^{2}\left(x^{2}+2 c x+c^{2}+y^{2}\right)=a^{4}+2 a^{2}(c x)+(c x)^{2} \\
& \Rightarrow a^{2} x^{2}+2 a^{2} c x+a^{2} c^{2}+a^{2} y^{2}=a^{4}+2 a^{2} c x+c^{2} x^{2} .
\end{aligned}
$$

Cancelando o termo $2 a^{2} c x$ em ambos os lados da equação acima, temos:

$a^{2} x^{2}+a^{2} c^{2}+a^{2} y^{2}=a^{4}+c^{2} x^{2}$

$\Rightarrow a^{2} x^{2}-c^{2} x^{2}+a^{2} y^{2}=a^{4}-a^{2} c^{2}$

$\Rightarrow x^{2}\left(a^{2}-c^{2}\right)+a^{2} y^{2}=a^{2}\left(a^{2}-c^{2}\right)$.

Como $a \neq c$, podemos dividir ambos os lados por $a^{2}\left(a^{2}-c^{2}\right)$ e ficamos com:

$$
\frac{x^{2}\left(a^{2}-c^{2}\right)}{a^{2}\left(a^{2}-c^{2}\right)}+\frac{a^{2} y^{2}}{a^{2}\left(a^{2}-c^{2}\right)}=\frac{a^{2}\left(a^{2}-c^{2}\right)}{a^{2}\left(a^{2}-c^{2}\right)} .
$$

Simplificando, obtemos:

$$
\frac{x^{2}}{a^{2}}+\frac{y^{2}}{a^{2}-c^{2}}=1 \text {. }
$$

Como $a>c, a^{2}-c^{2}>0$, então existe um número real $b=\sqrt{a^{2}-c^{2}}>0$ tal que $b^{2}=a^{2}-c^{2}$.

Se $P$ estiver no eixo $y$ positivo como na figura abaixo, então o triângulo $P O F_{1}$ é retângulo de catetos $P O$ e $O F_{1}$ e hipotenusa $P F 1$. Pelo teorema de Pitágoras, $\operatorname{dist}(P, O)^{2}+c^{2}=a^{2}$, ou seja, $\operatorname{dist}(P, O)^{2}=a^{2}-c^{2}$, logo $\operatorname{dist}(P, O)=b$ e $P=(0, b)$.

Usando $b$, chegamos na equação:

$$
\frac{x^{2}}{a^{2}}+\frac{y^{2}}{b^{2}}=1 \text {. }
$$




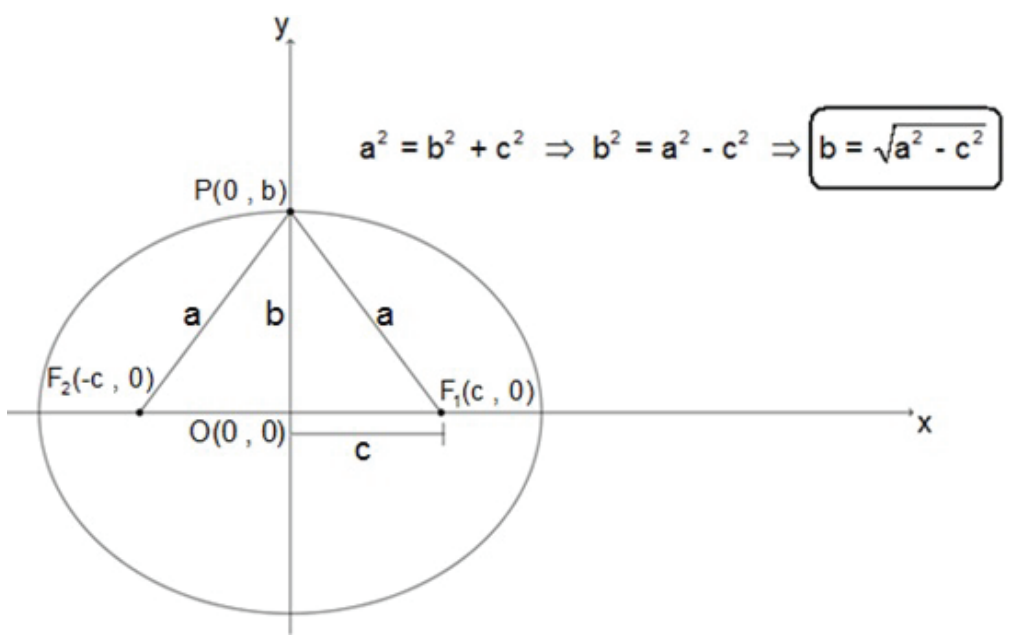

Figura 3.2: Elipse Propriedades 1

Reciprocamente, se as coordenadas de um ponto $P=(x, y)$ satisfazem a equação $\frac{x^{2}}{a^{2}}+\frac{y^{2}}{b^{2}}=1$ para $a \geq b>0$, então o ponto $P$ pertence à elipse de focos $F_{1}=(c, 0)$ e $F_{2}=(-c, 0)$ e constante $2 a$, onde $c=\sqrt{a^{2}-b^{2}}$. Para provar essa recíproca basta seguir as contas acima no caminho inverso tomando cuidado com a parte onde elevamos ao quadrado (veja no Apêndice A a demonstração com os detalhes).

Se $P=(x, y)$ é um ponto da elipse, então $-a \leq x \leq a$, pois $-\frac{y^{2}}{b^{2}} \leq 0 \mathrm{e}$ somando 1 dos dois lados, temos $\frac{x^{2}}{a^{2}}=1-\frac{y^{2}}{b^{2}} \leq 1$, o que implica que $x^{2} \leq a^{2}$, ou seja, $-a \leq x \leq a$. Observamos que $(-a, 0)$ e $(a, 0)$ são os pontos de interseção da elipse com o eixo $x$ conforme ilustrado na figura 3.3.

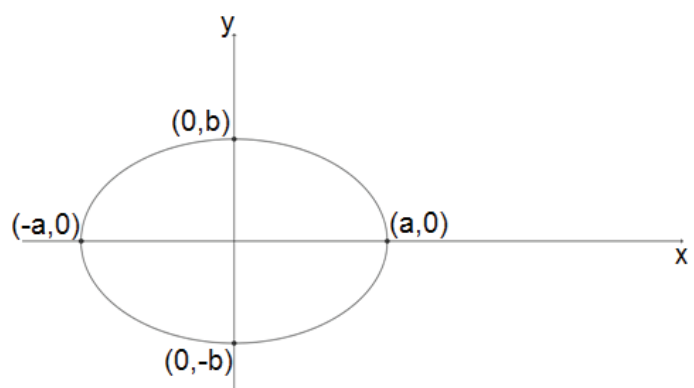

Figura 3.3: Elipse Propriedades 2

\section{2}

\section{Propriedade reflexiva da elipse}

Vamos agora mostrar a propriedade reflexiva da elipse. Ela nos diz que se uma reta passa por um dos focos da elipse, digamos $F_{1}$ e por um ponto $P$ 
da elipse, ela será refletida e passará pelo ponto $P$ e pelo outro foco da elipse $F_{2}$.

Observe que a elipse é simétrica em relação ao eixo $x$ e ao eixo $y$. Então, vamos restringir a análise ao caso em que $P$ pertence a parte da elipse que está no primeiro quadrante. Isto significa que estamos supondo $x \geq 0$ e $y \geq 0$, tais que $\frac{x^{2}}{a^{2}}+\frac{y^{2}}{b^{2}}=1$. Agora vamos definir a função cujo gráfico é a parte da elipse no primeiro quadrante e encontrar a equação da reta tangente em um ponto.

$$
\begin{aligned}
& y^{2}=b^{2}\left(1-\frac{x^{2}}{a^{2}}\right) \\
& \Rightarrow y=\sqrt{b^{2}\left(1-\frac{x^{2}}{a^{2}}\right)} \\
& \Rightarrow y=\sqrt{b^{2}} \sqrt{1-\frac{x^{2}}{a^{2}}} \\
& \Rightarrow y=|b| \sqrt{1-\frac{x^{2}}{a^{2}}} \\
& \Rightarrow y=|b| \sqrt{\frac{a^{2}-x^{2}}{a^{2}}} \\
& \Rightarrow y=|b| \frac{\sqrt{a^{2}-x^{2}}}{\sqrt{a^{2}}} \\
& \Rightarrow y=\frac{|b|}{|a|} \sqrt{a^{2}-x^{2}} .
\end{aligned}
$$

Como $a>0$ e $b>0$, temos $|a|=a$ e $|b|=b$. Logo:

$$
y=\frac{b}{a} \sqrt{a^{2}-x^{2}}
$$

A função

$$
\begin{aligned}
f:[0, a] & \longrightarrow \mathbb{R} \\
x & \longmapsto f(x)=\frac{b}{a} \sqrt{a^{2}-x^{2}}
\end{aligned}
$$

é derivável em $x \in[0, a)$ e sua derivada é:

$$
f^{\prime}(x)=\frac{b}{a}\left(\frac{-2 x}{2 \sqrt{a^{2}-x^{2}}}\right)=-\frac{b}{a} \frac{x}{\sqrt{a^{2}-x^{2}}} .
$$

Seja $P=\left(x_{0}, y_{0}\right)$ um ponto do gráfico de $f$ com $x_{0} \neq a$ e $t$ a reta tangente em $P$. A equação de $t$ é

$$
y-y_{0}=-\frac{b}{a} \frac{x_{0}}{\sqrt{a^{2}-x_{0}^{2}}}\left(x-x_{0}\right) .
$$

Seja $\alpha$ o ângulo formado pela reta que passa pelos pontos $F_{2}$ e $P$ e a reta $t$, e $\beta$ o ângulo formado pela reta que passa pelos pontos $F_{1}$ e $P$ e a reta $t$ tais que nem $\alpha$ e nem $\beta$ contenham o ângulo $\angle F_{2} P F_{1}$ e nem seu oposto com relação a $P$, como na figura abaixo. 


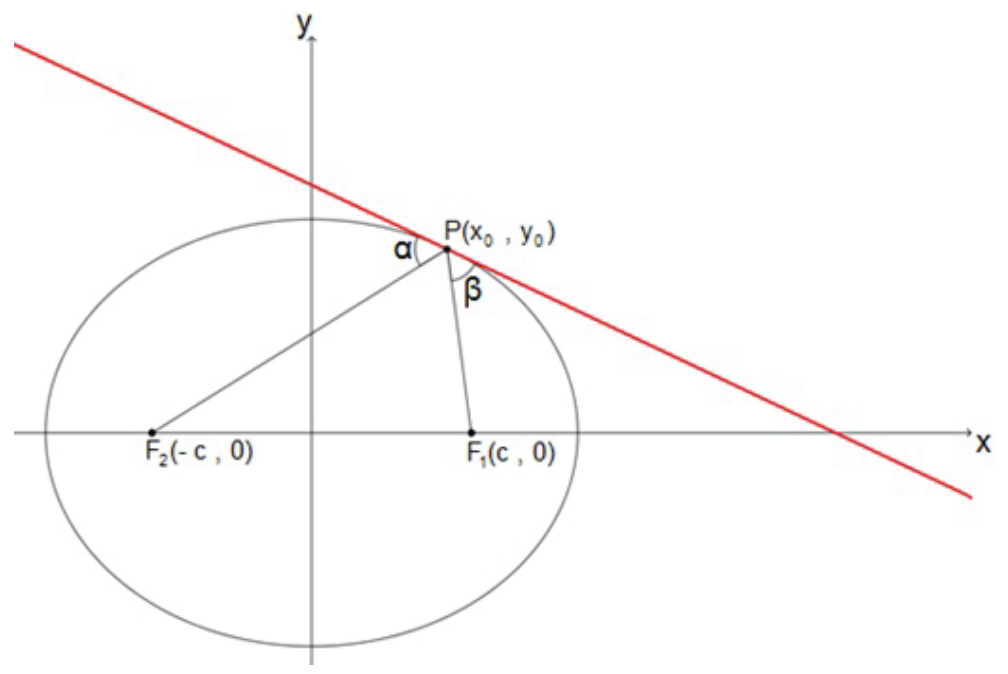

Figura 3.4: Propriedade Reflexiva Elipse

Para que essa definição de $\alpha$ e $\beta$ faça sentido é preciso mostrar que os dois focos sempre estão no mesmo semiplano definido por $t$. Equivalentemente, devemos mostrar que a primeira coordenada do ponto, digamos $U$, de interseção de $t$ com o eixo $x$, é maior que $a$, já que $-c<c<a$ :

Como $U$ está em $t$, temos

$0-y_{0}=-\frac{b}{a} \frac{x_{0}}{\sqrt{a^{2}-x_{0}^{2}}}\left(x-x_{0}\right)$.

Usando $y_{0}=\frac{b}{a} \sqrt{a^{2}-x_{0}^{2}}$ :

$0-\frac{b}{a} \sqrt{a^{2}-x_{0}^{2}}=-\frac{b}{a} \frac{x_{0}}{\sqrt{a^{2}-x_{0}^{2}}}\left(x-x_{0}\right)$

$\Rightarrow \sqrt{a^{2}-x_{0}^{2}}=\frac{x_{0}}{\sqrt{a^{2}-x_{0}^{2}}}\left(x-x_{0}\right)$

$\Rightarrow \frac{a^{2}-x_{0}^{2}}{x_{0}}=x-x_{0}$

$\Rightarrow \frac{a^{2}-x_{0}^{2}}{x_{0}}+x_{0}=x$

$\Rightarrow \frac{a^{2}-x_{0}^{2}+x_{0}^{2}}{x_{0}}=x$

$\Rightarrow x=\frac{a^{2}}{x_{0}}$.

Como $0<x_{0}<a$, vale que $a x_{0}<a^{2}$ e $a<\frac{a^{2}}{x_{0}}$.

Mostrar a propriedade reflexiva significa mostrar que $\alpha=\beta$. Primeiramente, iremos mostrar que os ângulos $\alpha$ e $\beta$ são agudos, e para isso basta mostrar que o ponto de interseção, digamos $Q$, da reta perpendicular à reta tangente que passa por $P=\left(x_{0}, y_{0}\right)$, digamos $s$, com o eixo $x$ está entre $F_{1}$ e $F_{2}$, pois com isso a semirreta que passa pelos pontos $P$ e $Q$ está entre as semirretas que passam pelos pontos $P$ e $F_{1}$ e por $P$ e $F_{2}$ como ilustrado na 
figura 3.5. A reta tangente $t$ é horizontal se e só se $f^{\prime}\left(x_{0}\right)=0$, ou seja, se e só se $x_{0}=0$, e neste caso o ponto $P$ está no eixo $y$, a reta $s$ é o eixo $y$ e $Q$ é a origem o que garante que $Q$ está entre os focos. Agora assumimos que $f^{\prime}\left(x_{0}\right) \neq 0$. Assim, a reta $s$ tem a seguinte equação:

$$
\begin{aligned}
& y-y_{0}=-\frac{1}{f^{\prime}\left(x_{0}\right)}\left(x-x_{0}\right) \\
& y-y_{0}=\frac{a}{b} \frac{\sqrt{a^{2}-x_{0}^{2}}}{x_{0}}\left(x-x_{0}\right) .
\end{aligned}
$$

Vamos agora determinar a interseção desta reta com o eixo $x$, ou seja, calcular a primeira coordenada de $Q$. Fazendo $y=0$, temos que:

$$
\begin{aligned}
& 0-y_{0}=\frac{a}{b} \frac{\sqrt{a^{2}-x_{0}^{2}}}{x_{0}}\left(x-x_{0}\right) \\
& \Rightarrow-y_{0}=\frac{a}{b} \frac{\sqrt{a^{2}-x_{0}^{2}}}{x_{0}}\left(x-x_{0}\right) \\
& \Rightarrow-\frac{b}{a} \sqrt{a^{2}-x_{0}^{2}}=\frac{a}{b} \frac{\sqrt{a^{2}-x_{0}^{2}}}{x_{0}}\left(x-x_{0}\right)
\end{aligned}
$$

Como estamos supondo $x_{0} \neq a$, temos

$$
\begin{aligned}
& -\frac{b}{a}=\frac{a}{b} \frac{1}{x_{0}}\left(x-x_{0}\right) \\
& \Rightarrow-\frac{b^{2} x_{0}}{a^{2}}=x-x_{0} \\
& \Rightarrow x=x_{0}-\frac{b^{2} x_{0}}{a^{2}} \\
& \Rightarrow x=\frac{a^{2} x_{0}-b^{2} x_{0}}{a^{2}} \\
& \Rightarrow x=\frac{\left(a^{2}-b^{2}\right) x_{0}}{a^{2}} .
\end{aligned}
$$

Como $a^{2}-b^{2}=c^{2}$, temos que $x=\frac{c^{2}}{a^{2}} x_{0}$. Então o ponto de interseção da reta perpendicular à reta tangente que passa por $P=\left(x_{0}, y_{0}\right)$ com o eixo $x$ será $Q=\left(\frac{c^{2}}{a^{2}} x_{0}, 0\right)$. Temos que mostrar que $-c<\frac{c^{2}}{a^{2}} x_{0}<c$ como aparece na figura abaixo 3.5 .

Como estamos supondo $x_{0}>0$ e sabemos que $-c$ é negativo, temos $\frac{c^{2}}{a^{2}} x_{0}>0>-c \therefore \frac{c^{2}}{a^{2}} x_{0}>-c$.

Lembrando que $0<c<a, 0<x_{0}<a$, temos $c x_{0}<a^{2}$. Multiplicando os dois lados por $c$, que é positivo, obtemos $c^{2} x_{0}<c a^{2}$ e finalmente $\frac{c^{2} x_{0}}{a^{2}}<c$.

Portanto:

$$
-c<\frac{c^{2}}{a^{2}} x_{0}<c
$$




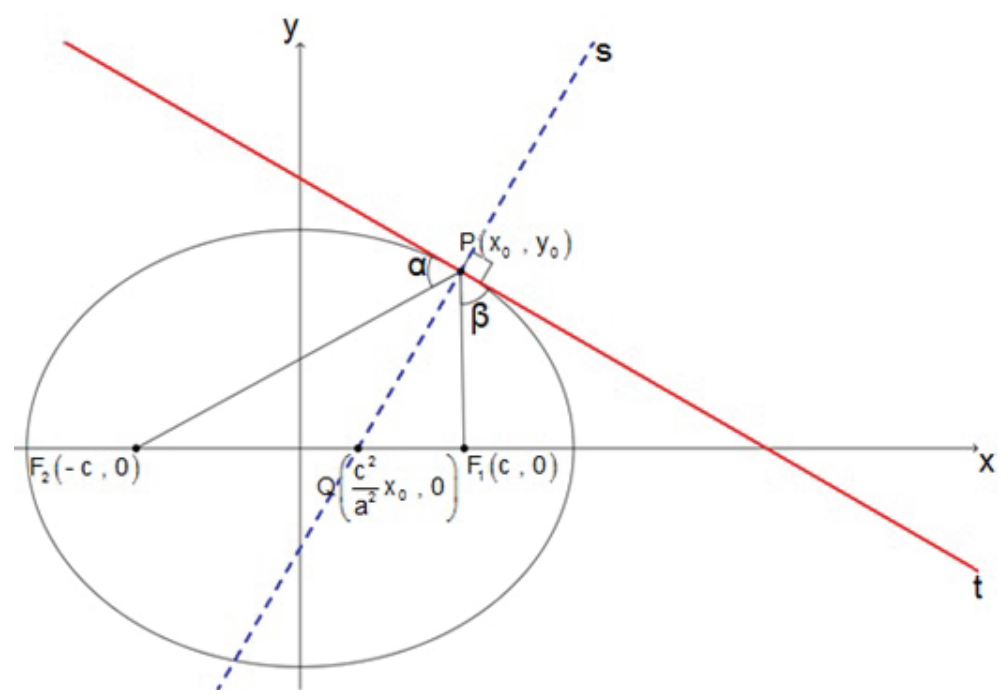

Figura 3.5: Elipse Reta Ortogonal 1

Com isso, a reta $s$ (perpendicular à reta tangente $t$ por $P$ ) passa entre as retas que passam pelos pontos $P=\left(x_{0}, y_{0}\right)$ e $F_{1}=(c, 0)$ e pelos pontos $P=\left(x_{0}, y_{0}\right)$ e $F_{2}=(-c, 0)$. Provamos com isso que os ângulos $\alpha$ e $\beta$ são agudos.

Agora, vamos mostrar que $\alpha=\beta$. Para isso, iremos demonstrar que $\operatorname{tg} \alpha=\operatorname{tg} \beta$.

A demonstração a seguir envolve contas com o coeficiente angular da reta que passa por $P$ e $F_{1}$, mas se $x_{0}=c$ a essa reta é vertical, por isso vamos supor no momento $x_{0} \neq c$. Começamos com as seguintes considerações:

$m_{1}$ : coeficiente angular da reta que passa pelos pontos $F_{1}=(c, 0)$ e $P=\left(x_{0}, y_{0}\right)$.

$$
m_{1}=\frac{y_{0}-0}{x_{0}-c}=\frac{y_{0}}{x_{0}-c} .
$$

$m_{2}$ : coeficiente angular da reta que passa pelos pontos $F_{2}=(-c, 0)$ e $P=\left(x_{0}, y_{0}\right)$.

$$
m_{2}=\frac{y_{0}-0}{x_{0}-(-c)}=\frac{y_{0}}{x_{0}+c} \text {. }
$$

Observe agora a figura a seguir:

Podemos concluir que:

$$
\begin{aligned}
& m_{1}=\operatorname{tg} \theta_{1}, \\
& m_{2}=\operatorname{tg} \theta_{2} \quad \text { e } \\
& f^{\prime}\left(x_{0}\right)=\operatorname{tg} \theta \Rightarrow \operatorname{tg} \theta=-\frac{b}{a} \frac{x_{0}}{\sqrt{a^{2}-x_{0}^{2}}} .
\end{aligned}
$$

Além disso:

$$
\begin{aligned}
& \alpha=\theta_{2}+\pi-\theta \Rightarrow \operatorname{tg} \alpha=\operatorname{tg}\left(\theta_{2}+\pi-\theta\right) \Rightarrow \operatorname{tg} \alpha=\operatorname{tg}\left(\theta_{2}-\theta\right) \Rightarrow \operatorname{tg} \alpha= \\
& \frac{\operatorname{tg} \theta_{2}-\operatorname{tg} \theta}{1+\left(\operatorname{tg} \theta_{2}\right)(\operatorname{tg} \theta)} \text {. }
\end{aligned}
$$




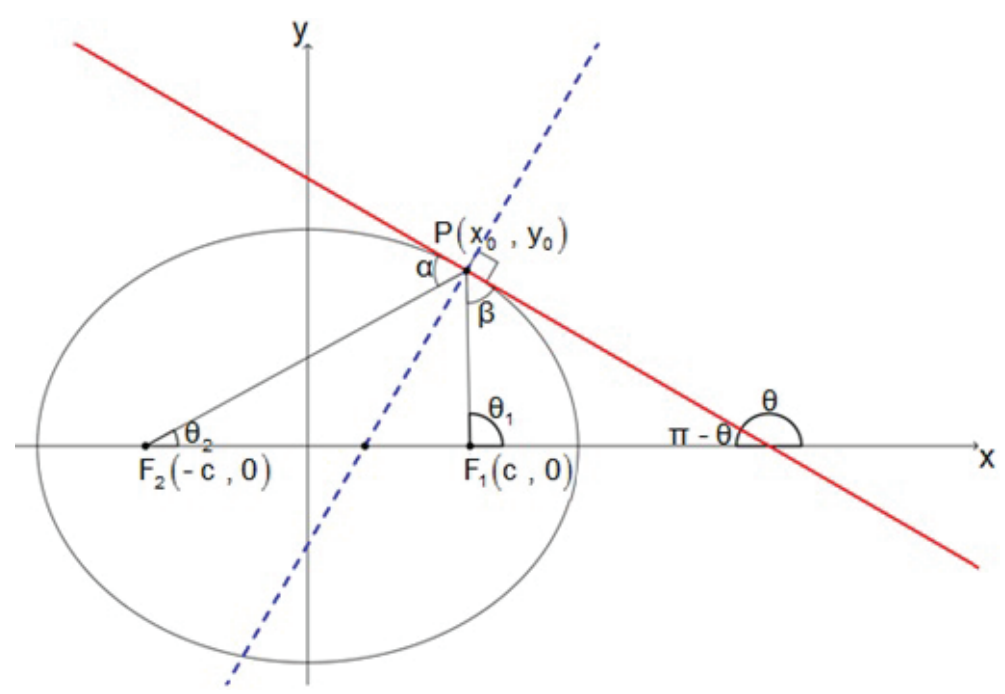

Figura 3.6: Elipse Reta Ortogonal 2

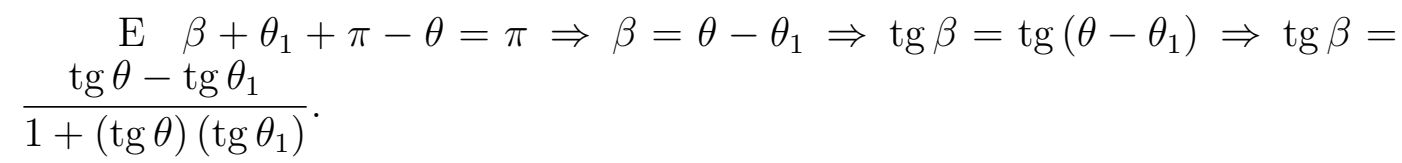

$\operatorname{tg} \alpha=\frac{\operatorname{tg} \theta_{2}-\operatorname{tg} \theta}{1+\left(\operatorname{tg} \theta_{2}\right)(\operatorname{tg} \theta)} \Rightarrow \operatorname{tg} \alpha=\frac{m_{2}-f^{\prime}\left(x_{0}\right)}{1+m_{2} f^{\prime}\left(x_{0}\right)}$

$\mathrm{E} \operatorname{tg} \beta=\frac{\operatorname{tg} \theta-\operatorname{tg} \theta_{1}}{1+(\operatorname{tg} \theta)\left(\operatorname{tg} \theta_{1}\right)} \Rightarrow \operatorname{tg} \beta=\frac{f^{\prime}\left(x_{0}\right)-m_{1}}{1+f^{\prime}\left(x_{0}\right) m_{1}}$.

Desenvolvendo os cálculos para $\operatorname{tg} \alpha$ :

$\operatorname{tg} \alpha=\frac{\left(\frac{y_{0}}{x_{0}+c}\right)-\left(-\frac{b}{a} \frac{x_{0}}{\sqrt{a^{2}-x_{0}^{2}}}\right)}{1+\left(\frac{y_{0}}{x_{0}+c}\right)\left(-\frac{b}{a} \frac{x_{0}}{\sqrt{a^{2}-x_{0}^{2}}}\right)} \Rightarrow \operatorname{tg} \alpha=\frac{\frac{b}{a} \frac{\sqrt{a^{2}-x_{0}{ }^{2}}}{x_{0}+c}+\frac{b}{a} \frac{x_{0}}{\sqrt{a^{2}-x_{0}^{2}}}}{1-\left(\frac{b}{a} \frac{\sqrt{a^{2}-x_{0}{ }^{2}}}{x_{0}+c}\right)\left(\frac{b}{a} \frac{x_{0}}{\sqrt{a^{2}-x_{0}^{2}}}\right)}$

$\Rightarrow \operatorname{tg} \alpha=\frac{\frac{b}{a} \frac{x_{0}\left(x_{0}+c\right)+\left(a^{2}-x_{0}^{2}\right)}{\left(x_{0}+c\right) \sqrt{a^{2}-x_{0}^{2}}}}{\frac{a^{2}\left(x_{0}+c\right)-b^{2} x_{0}}{a^{2}\left(x_{0}+c\right)}} \Rightarrow \operatorname{tg} \alpha=\frac{a b\left(x_{0}{ }^{2}+x_{0} c+a^{2}-x_{0}^{2}\right)}{\left(a^{2} x_{0}+a^{2} c-b^{2} x_{0}\right) \sqrt{a^{2}-x_{0}^{2}}}$

$\Rightarrow \operatorname{tg} \alpha=\frac{a b\left(x_{0} c+a^{2}\right)}{\left(x_{0}\left(a^{2}-b^{2}\right)+a^{2} c\right) \sqrt{a^{2}-x_{0}^{2}}}$.

Como $a^{2}-b^{2}=c^{2}$, temos que:

$\operatorname{tg} \alpha=\frac{a b\left(x_{0} c+a^{2}\right)}{\left(x_{0} c^{2}+a^{2} c\right) \sqrt{a^{2}-x_{0}^{2}}}=\frac{a b\left(x_{0} c+a^{2}\right)}{c\left(x_{0} c+a^{2}\right) \sqrt{a^{2}-x_{0}^{2}}}=$
$\frac{a b\left(x_{0} c+a^{2}\right)}{c\left(x_{0} c+a^{2}\right) \sqrt{a^{2}-x_{0}^{2}}}$.

Cancelando o termo $x_{0} c+a^{2}$, temos que: 


$$
\operatorname{tg} \alpha=\frac{a b}{c \sqrt{a^{2}-x_{0}^{2}}} .
$$

Vamos agora desenvolver os cálculos para obter $\operatorname{tg} \beta$. De fato:

$$
\begin{aligned}
& \operatorname{tg} \beta=\frac{\left(-\frac{b}{a} \frac{x_{0}}{\sqrt{a^{2}-x_{0}^{2}}}\right)-\left(\frac{y_{0}}{x_{0}-c}\right)}{1+\left(-\frac{b}{a} \frac{x_{0}}{\sqrt{a^{2}-x_{0}^{2}}}\right)\left(\frac{y_{0}}{x_{0}-c}\right)} \Rightarrow \operatorname{tg} \beta=\frac{-\frac{b}{a} \frac{x_{0}}{\sqrt{a^{2}-x_{0}^{2}}}-\frac{b}{a} \frac{\sqrt{a^{2}-x_{0}{ }^{2}}}{x_{0}-c}}{1-\frac{b}{a} \frac{x_{0}}{\sqrt{a^{2}-x_{0}^{2}}}\left(\frac{b}{a} \frac{\sqrt{a^{2}-x_{0}^{2}}}{x_{0}-c}\right)} \\
& \Rightarrow \operatorname{tg} \beta=\frac{-\frac{b}{a} \frac{x_{0}\left(x_{0}-c\right)+\left(a^{2}-x_{0}^{2}\right)}{\left(x_{0}-c\right) \sqrt{a^{2}-x_{0}{ }^{2}}}}{\frac{a^{2}\left(x_{0}-c\right)-b^{2} x_{0}}{a^{2}\left(x_{0}-c\right)}} \Rightarrow \operatorname{tg} \beta=\frac{-a b\left(x_{0}^{2}-x_{0} c+a^{2}-x_{0}^{2}\right)}{\left(a^{2} x_{0}-a^{2} c-b^{2} x_{0}\right) \sqrt{a^{2}-x_{0}^{2}}} \\
& \Rightarrow \operatorname{tg} \beta=\frac{-a b\left(-x_{0} c+a^{2}\right)}{\left(x_{0}\left(a^{2}-b^{2}\right)-a^{2} c\right) \sqrt{a^{2}-x_{0}^{2}}} .
\end{aligned}
$$

Como $a^{2}-b^{2}=c^{2}$, temos que:

$\operatorname{tg} \beta=\frac{a b\left(x_{0} c-a^{2}\right)}{\left(x_{0} c^{2}-a^{2} c\right) \sqrt{a^{2}-x_{0}^{2}}}=\frac{a b\left(x_{0} c-a^{2}\right)}{c\left(x_{0} c-a^{2}\right) \sqrt{a^{2}-x_{0}^{2}}}=$ $\frac{a b\left(x_{0} c-a^{2}\right)}{c\left(x_{0} c-a^{2}\right) \sqrt{a^{2}-x_{0}^{2}}}$.

$$
\Rightarrow \operatorname{tg} \beta=\frac{a b\left(x_{0} c-a^{2}\right)}{c\left(x_{0} c-a^{2}\right) \sqrt{a^{2}-x_{0}^{2}}} .
$$

Provamos anteriormente que $\frac{c^{2}}{a^{2}} x_{0}<c$. Como $c>0$, temos que:

$\frac{c}{a^{2}} x_{0}<1$.

Como $a>0$, temos que:

$c x_{0}<a^{2}$

$c x_{0}-a^{2}<0$.

Logo, $c x_{0}-a^{2} \neq 0$ e então podemos cancelar o termo $x_{0} c-a^{2}$, e temos que:

$$
\operatorname{tg} \beta=\frac{a b}{c \sqrt{a^{2}-x_{0}^{2}}} .
$$

Com isso, concluímos que $\operatorname{tg} \alpha=\operatorname{tg} \beta$ no caso em que $x_{0} \neq c$ e $x_{0} \neq a$.

Vejamos o caso $x_{0}=c$, ou seja, caso $P=\left(c, \frac{b}{a} \sqrt{a^{2}-c^{2}}\right)=\left(c, \frac{b^{2}}{a}\right)$. As contas feitas acima pata $\operatorname{tg} \alpha$ continuam valendo, ou seja, $\operatorname{tg} \alpha=\frac{a b}{c \sqrt{a^{2}-x_{0}^{2}}}$.

Substituindo $x_{0}$ por $c$ e $\sqrt{a^{2}-c^{2}}$ por $b$, temos $\operatorname{tg} \alpha=\frac{a b}{c \sqrt{a^{2}-c^{2}}}=\frac{a b}{b}=\frac{a}{c}$.

Agora para calcular tg $\beta$ lembramos que $P F_{1} U$ é retângulo: 


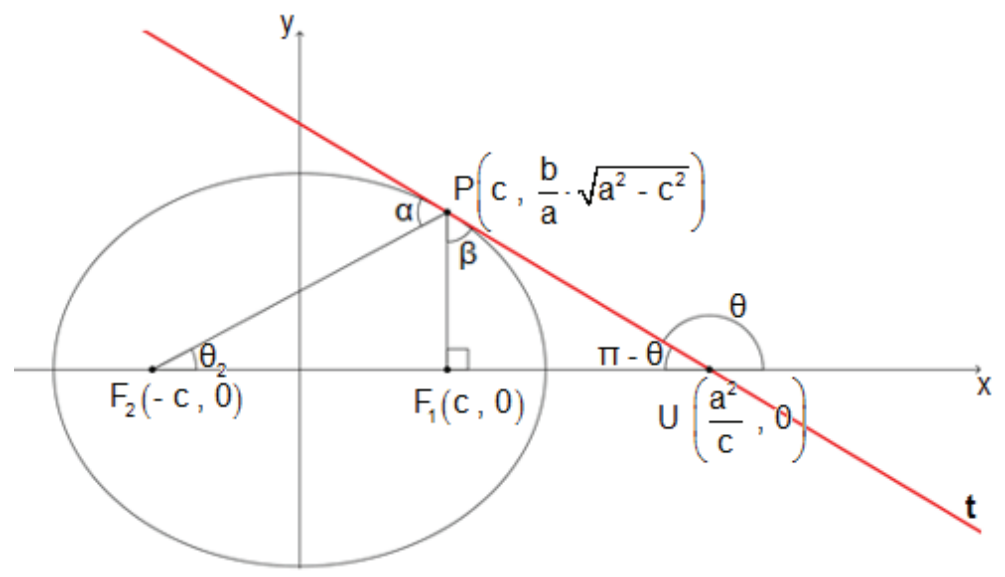

Figura 3.7: Elipse ângulo reto

$$
\operatorname{tg} \beta=\frac{\operatorname{dist}\left(F_{1}, U\right)}{\operatorname{dist}\left(F_{1}, P\right)}=\frac{\frac{a^{2}}{c}-c}{\frac{b^{2}}{a}}=\frac{a^{2}-c^{2}}{c} \cdot \frac{a}{b^{2}}=\frac{a}{c} .
$$

Concluímos assim que $\operatorname{tg} \alpha=\operatorname{tg} \beta$ também no caso em que $x_{0}=c$. Como $\alpha$ e $\beta$ são ângulos agudos segue que:

$$
\alpha=\beta \text {. }
$$

Resta apenas discutirmos o caso em que $x_{0}=a$, ou seja, $P=(a, 0)$. Nesse caso a propriedade reflexiva é satisfeita trivialmente, pois a reta que passa por $P$ e $F_{1}$ e a reta que passa por $P$ e $F_{2}$ são ambas iguais ao eixo $x$, e a reta tangente à elipse em $P$ é vertical de equação $x=a$. 


\section{Hipérbole}

Uma hipérbole é o conjunto dos pontos do plano tais que o valor absoluto da diferença das distâncias a dois pontos fixos, ditos focos, é constante e essa constante é menor que a distância entre os focos.

\section{1}

\section{Equação cartesiana da hipérbole}

Sejam $F_{1}$ e $F_{2}$ dois pontos distintos no plano e $a>0$ tal que $\operatorname{dist}\left(F_{1}, F_{2}\right)>$ $2 a$. Vamos deduzir a equação cartesiana da hipérbole com focos $F_{1}$ e $F_{2}$ e constante $2 a$. Para facilitar as contas, o eixo $x$ será a reta que passa pelos focos e o eixo $y$ a reta perpendicular que passa pelo ponto médio dos focos. Com isso, podemos escrever $F_{1}=(c, 0)$ e $F_{2}=(-c, 0)$, onde $2 c$ é a distância entre os focos.

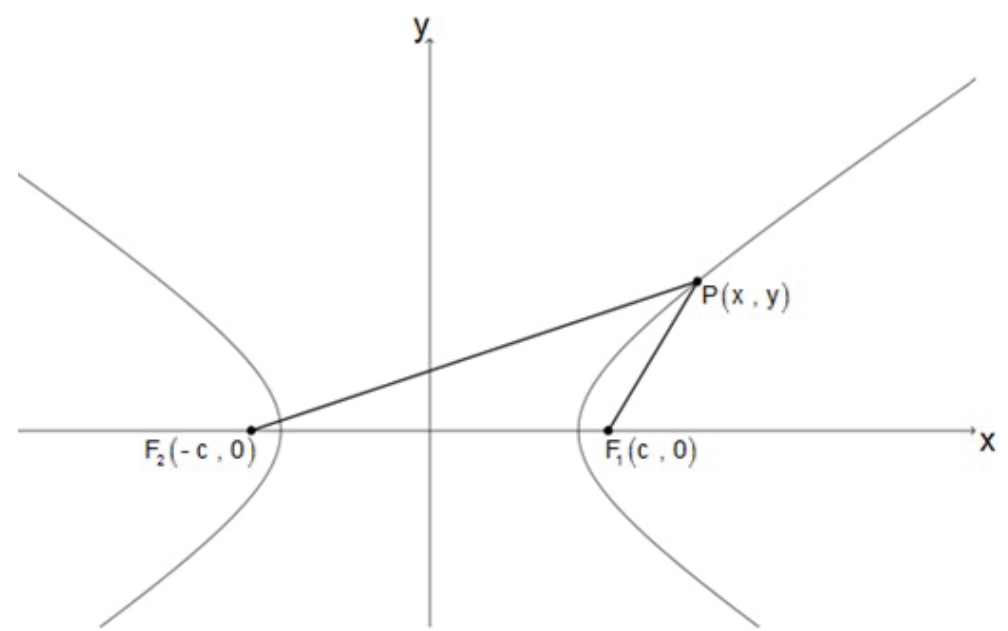

Figura 4.1: Hipérbole Definição

Considere $P=(x, y)$ um ponto pertencente à hipérbole.

Foi observado que, pela desigualdade triangular, que $2 a<2 c$, ou seja, $a<c$. Vejamos isso com detalhes: Pela desigualdade triangular temos $\operatorname{dist}\left(P, F_{1}\right)+\operatorname{dist}\left(F_{2}, F_{1}\right)>\operatorname{dist}\left(P, F_{2}\right) \quad$ e $\operatorname{dist}\left(P, F_{2}\right)+\operatorname{dist}\left(F_{2}, F_{1}\right)>$ $\operatorname{dist}\left(P, F_{1}\right)$. Como $\operatorname{dist}\left(F_{2}, F_{1}\right)=2 c, \operatorname{temos} \operatorname{dist}\left(P, F_{1}\right)+2 c>\operatorname{dist}\left(P, F_{2}\right)$ e $\operatorname{dist}\left(P, F_{2}\right)+2 c>\operatorname{dist}\left(P, F_{1}\right)$, ou ainda, $2 c>\operatorname{dist}\left(P, F_{2}\right)-\operatorname{dist}\left(P, F_{1}\right) \quad$ e $2 c>\operatorname{dist}\left(P, F_{1}\right)-\operatorname{dist}\left(P, F_{2}\right)$. Se $\operatorname{dist}\left(P, F_{2}\right)>\operatorname{dist}\left(P, F_{1}\right)$, então $\operatorname{dist}\left(P, F_{2}\right)-$ $\operatorname{dist}\left(P, F_{1}\right)=2 a$ e a primeira desigualdade acima garante que $2 c>2 a$. Se 
$\operatorname{dist}\left(P, F_{1}\right)>\operatorname{dist}\left(P, F_{2}\right)$, então $\operatorname{dist}\left(P, F_{1}\right)-\operatorname{dist}\left(P, F_{2}\right)=2 a$ e a segunda desigualdade acima garante que $2 c>2 a$. Finalmente $c>a$.

Vamos encontrar agora a equação da hipérbole. Pela definição, sabemos que

$$
\left|\operatorname{dist}\left(P, F_{1}\right)-\operatorname{dist}\left(P, F_{2}\right)\right|=2 a .
$$

Efetuando os cálculos:

$\left|\operatorname{dist}\left(P, F_{1}\right)-\operatorname{dist}\left(P, F_{2}\right)\right|=2 a$

$\sqrt{(x-c)^{2}+(y-0)^{2}}-\sqrt{(x-(-c))^{2}+(y-0)^{2}}= \pm 2 a$

$\sqrt{(x-c)^{2}+y^{2}}-\sqrt{(x+c)^{2}+y^{2}}= \pm 2 a$

$\sqrt{(x-c)^{2}+y^{2}}=\sqrt{(x+c)^{2}+y^{2}} \pm 2 a$.

Elevando ambos os membros ao quadrado, temos:

$$
\begin{aligned}
& \left(\sqrt{(x-c)^{2}+y^{2}}\right)^{2}=\left(\sqrt{(x+c)^{2}+y^{2}} \pm 2 a\right)^{2} \\
& (x-c)^{2}+y^{2}=(x+c)^{2}+y^{2} \pm 4 a \sqrt{(x+c)^{2}+y^{2}}+(2 a)^{2} \\
& x^{2}-2 x c+c^{2}+y^{2}=x^{2}+2 x c+c^{2}+y^{2} \pm 4 a \sqrt{(x+c)^{2}+y^{2}}+4 a^{2} \\
& \pm 4 a \sqrt{(x+c)^{2}+y^{2}}=-4 a^{2}-4 x c \\
& \pm a \sqrt{(x+c)^{2}+y^{2}}=-a^{2}-x c .
\end{aligned}
$$

Elevando ambos os membros ao quadrado, temos que:

$$
\begin{aligned}
& \left( \pm a \sqrt{(x+c)^{2}+y^{2}}\right)^{2}=\left(-a^{2}-x c\right)^{2} \\
& ( \pm a)^{2}\left((x+c)^{2}+y^{2}\right)=a^{4}+2 a^{2} x c+c^{2} x^{2} \\
& a^{2}\left(x^{2}+2 x c+c^{2}+y^{2}\right)=a^{4}+2 a^{2} x c+c^{2} x^{2} \\
& a^{2} x^{2}+2 a^{2} x c+a^{2} c^{2}+a^{2} y^{2}=a^{4}+2 a^{2} x c+c^{2} x^{2} .
\end{aligned}
$$

Cancelando o termo $2 a^{2} x c$, temos que:

$a^{2} x^{2}+a^{2} c^{2}+a^{2} y^{2}=a^{4}+c^{2} x^{2}$

$a^{2} x^{2}-c^{2} x^{2}+a^{2} y^{2}=a^{4}-a^{2} c^{2}$

$x^{2}\left(a^{2}-c^{2}\right)+a^{2} y^{2}=a^{2}\left(a^{2}-c^{2}\right)$.

Lembrando que $a \neq c$, pois $a<c$, podemos dividir ambos os lados por $a^{2}\left(a^{2}-c^{2}\right)$, temos que:

$$
\frac{x^{2}\left(a^{2}-c^{2}\right)}{a^{2}\left(a^{2}-c^{2}\right)}+\frac{a^{2} y^{2}}{a^{2}\left(a^{2}-c^{2}\right)}=\frac{a^{2}\left(a^{2}-c^{2}\right)}{a^{2}\left(a^{2}-c^{2}\right)} .
$$

Assim

$$
\frac{x^{2}}{a^{2}}+\frac{y^{2}}{a^{2}-c^{2}}=1 \text {. }
$$

Agora observe a figura 4.2 abaixo.

Como $a>0, c>0$ e $a<c$, temos $a^{2}<c^{2} \therefore a^{2}-c^{2}<0$ e segue daí que existe um número real $b>0$ tal que $a^{2}-c^{2}=-b^{2}$. Logo:

$$
\frac{x^{2}}{a^{2}}+\frac{y^{2}}{-b^{2}}=1 \text {. }
$$




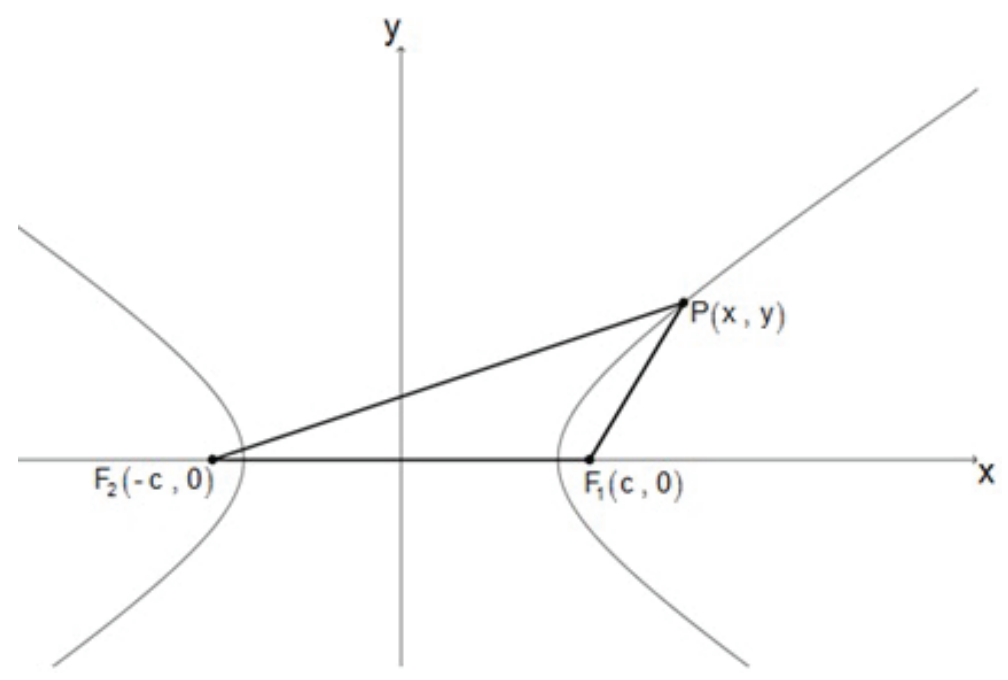

Figura 4.2: Hipérbole Propriedades

Ou seja,

$$
\frac{x^{2}}{a^{2}}-\frac{y^{2}}{b^{2}}=1
$$

Reciprocamente, se as coordenadas de um ponto $P=(x, y)$ satisfazem a equação $\frac{x^{2}}{a^{2}}-\frac{y^{2}}{b^{2}}=1$ para $a>0$ e $b>0$, então o ponto $P$ pertence à hipérbole de focos $F_{1}=(c, 0)$ e $F_{2}=(-c, 0)$ e constante $2 a$, onde $c=\sqrt{a^{2}+b^{2}}$. Para provar essa recíproca basta seguir as contas acima no caminho inverso tomando cuidado com a parte onde elevamos ao quadrado (veja no Apêndice $\mathrm{B}$ a demonstração com os detalhes).

Se $P=(x, y)$ é um ponto da hipérbole, então $x \leq-a$ ou $a \leq x$, pois $\frac{y^{2}}{b^{2}} \geq 0$ e somando-se 1 aos dois lados, temos $\frac{x^{2}}{a^{2}}=1+\frac{y^{2}}{b^{2}} \geq 1$, o que implica que $x^{2} \geq a^{2}$, ou seja, $x \leq-a$ ou $a \leq x$. Observamos que $(-a, 0)$ e $(a, 0)$ são os pontos de interseção da hipérbole com o eixo $x$.

\section{2}

\section{Propriedade reflexiva da hipérbole}

Vamos agora mostrar a propriedade reflexiva da hipérbole. Ela nos diz que se uma reta passa por um dos focos da hipérbole, digamos $F_{2}=(-c, 0)$, e por um ponto $P=\left(x_{0}, y_{0}\right)$ da hipérbole, ela será refletida e passará pelo ponto $P=\left(x_{0}, y_{0}\right)$ e pelo outro foco da hipérbole $F_{1}=(c, 0)$.

Primeiramente, considere um ponto $P=\left(x_{0}, y_{0}\right)$ qualquer da hipérbole de focos $F_{1}=(c, 0)$ e $F_{2}=(-c, 0)$.

Observe que a hipérbole é simétrica em relação ao eixo $x$ e ao eixo 
$y$. Então, vamos restringir a análise ao caso em que $P$ pertence à parte da hipérbole que está no primeiro quadrante. Isto significa que estamos supondo $x \geq a$ e $y \geq 0$, tais que $\frac{x^{2}}{a^{2}}-\frac{y^{2}}{b^{2}}=1$. Agora vamos definir a função cujo gráfico é a parte da hipérbole no primeiro quadrante e encontrar a equação da reta tangente em um ponto.

$$
\begin{aligned}
& \frac{x^{2}}{a^{2}}-\frac{y^{2}}{b^{2}}=1 \\
& \Rightarrow \frac{y^{2}}{b^{2}}=\frac{x^{2}}{a^{2}}-1 \\
& \Rightarrow \frac{y^{2}}{b^{2}}=\frac{x^{2}-a^{2}}{a^{2}} \\
& \Rightarrow y^{2}=\frac{b^{2}\left(x^{2}-a^{2}\right)}{a^{2}} \\
& \Rightarrow y=\sqrt{\frac{b^{2}\left(x^{2}-a^{2}\right)}{a^{2}}} \\
& \Rightarrow y=\frac{|b| \sqrt{x^{2}-a^{2}}}{|a|} \\
& \text { Como } a>0 \text { e } b>0,|a|=a \text { e }|b|=b . \text { Logo: } \\
& \Rightarrow=\frac{b}{a} \sqrt{x^{2}-a^{2}}
\end{aligned}
$$

A função

$$
\begin{aligned}
f:[a, \infty) & \longrightarrow \mathbb{R} \\
x & \longmapsto f(x)=\frac{b}{a} \sqrt{x^{2}-a^{2}}
\end{aligned}
$$

é derivável em $x \in(a, \infty)$ e sua derivada em cada ponto $x$ é:

$$
f^{\prime}(x)=\frac{b}{a}\left(\frac{2 x}{2 \sqrt{x^{2}-a^{2}}}\right)=\frac{b}{a} \frac{x}{\sqrt{x^{2}-a^{2}}} .
$$

Seja $P=\left(x_{0}, y_{0}\right)$ um ponto do gráfico de $f$ com $x_{0} \neq a$ e $t$ a reta tangente à hipérbole em $P$. A equação de $t$ é

$$
y-y_{0}=\frac{b}{a} \frac{x_{0}}{\sqrt{x_{0}^{2}-a^{2}}}\left(x-x_{0}\right)
$$

Seja $\alpha$ o ângulo formado pela reta que passa pelos pontos $F_{2}$ e $P$ e a reta $t$, e $\beta$ o ângulo formado pela reta que passa pelos pontos $F_{1}$ e $P$ e a reta $t$ tais que $\alpha$ e $\beta$ estão contidos em $\angle F_{2} P F_{1}$ e seu oposto com relação a $P$ como na figura abaixo.

Para que essa definição de $\alpha$ e $\beta$ faça sentido é preciso mostrar que os dois focos sempre estão em semiplanos, definido por $t$, diferentes. Equivalentemente, devemos mostrar que a primeira coordenada do ponto, digamos $Q$, de interseção de $t$ com o eixo $x$, está entre $-c$ e $c$.

Como $Q$ está em $t$, temos 


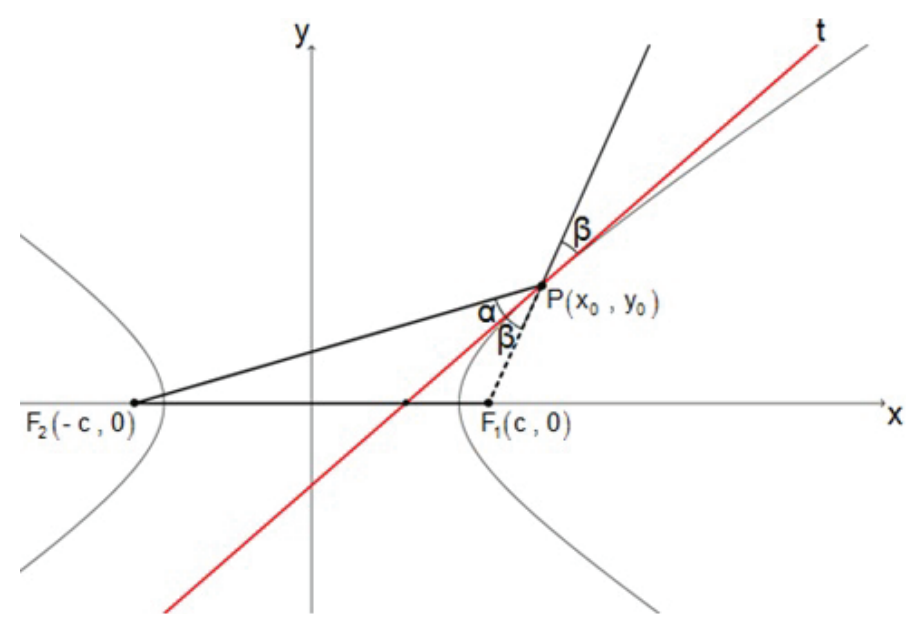

Figura 4.3: Propriedade Reflexiva da Hipérbole

$0-y_{0}=\frac{b}{a} \frac{x_{0}}{\sqrt{x_{0}^{2}-a^{2}}}\left(x-x_{0}\right)$.

Usando $y_{0}=\frac{b}{a} \sqrt{x_{0}^{2}-a^{2}}$ :

$0-\frac{b}{a} \sqrt{x_{0}^{2}-a^{2}}=\frac{b}{a} \frac{x_{0}}{\sqrt{x_{0}^{2}-a^{2}}}\left(x-x_{0}\right)$

$\Rightarrow-\sqrt{x_{0}^{2}-a^{2}}=\frac{x_{0}}{\sqrt{x_{0}^{2}-a^{2}}}\left(x-x_{0}\right)$

$\Rightarrow \frac{-\left(x_{0}^{2}-a^{2}\right)}{x_{0}}=x-x_{0}$

$\Rightarrow \frac{a^{2}-x_{0}^{2}}{x_{0}}+x_{0}=x$

$\Rightarrow \frac{a^{2}-x_{0}^{2}}{x_{0}}+x_{0}=x$

$\Rightarrow \frac{a^{2}-x_{0}^{2}+x_{0}^{2}}{x_{0}}=x$

$\Rightarrow x=\frac{a^{2}}{x_{0}}$.

Como $x_{0}>a$, vale que $a x_{0}>a^{2}$ e $a>\frac{a^{2}}{x_{0}}$. Lembrando que $-c<0$ e $a<c$, chegamos a $-c<0<\frac{a^{2}}{x_{0}}<a<c$.

Mostrar a propriedade reflexiva significa mostrar que $\alpha=\beta$. Primeiramente, iremos mostrar que os ângulos $\alpha$ e $\beta$ são agudos. Para isso, vamos fazer as seguintes considerações:

$u$ : reta que passa pelos pontos $F_{1}$ e $P$.

$v$ : reta que passa pelos pontos $F_{2}$ e $P$.

$t$ : reta tangente à hipérbole no ponto $P$.

$s:$ reta perpendicular à reta t no ponto $P$.

$W=t \cap\left\{(x, y) \in \mathbb{R}^{2} \mid x=0\right\}$ 


$$
\begin{aligned}
& Q=t \cap\left\{(x, y) \in \mathbb{R}^{2} \mid y=0\right\} \text { (como definido anteriormente) } \\
& T=s \cap\left\{(x, y) \in \mathbb{R}^{2} \mid x=0\right\} \\
& U=s \cap\left\{(x, y) \in \mathbb{R}^{2} \mid y=0\right\} \\
& M=v \cap\left\{(x, y) \in \mathbb{R}^{2} \mid x=0\right\}
\end{aligned}
$$

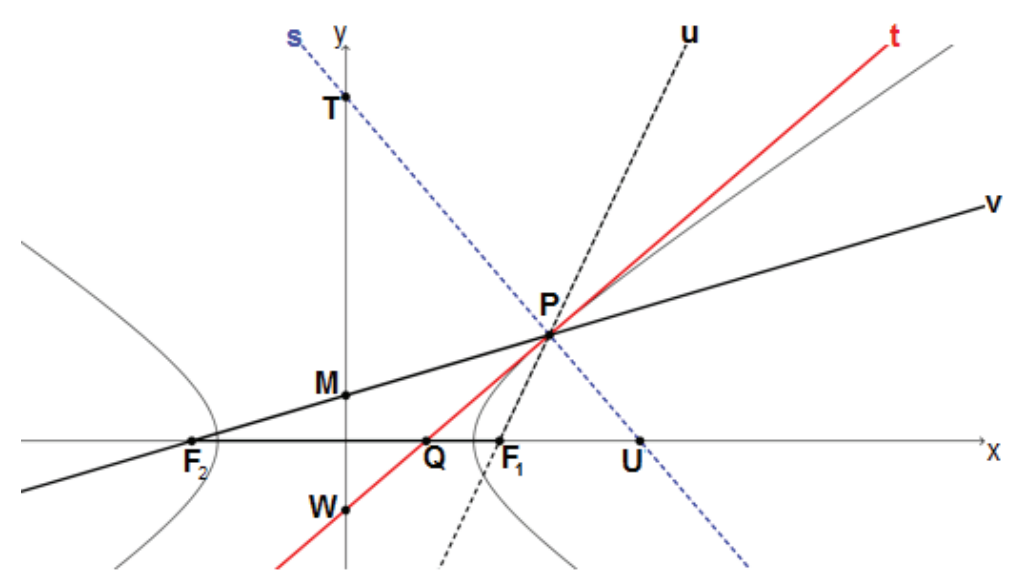

Figura 4.4: Hipérbole Pontos

Para mostrar que $\alpha$ é um ângulo agudo, basta mostrar que $M$ está entre $W$ e T. E para mostrar que $\beta$ é um ângulo agudo, basta mostrar que $F_{1}$ está entre $Q$ e $U$.

Equação da reta $t$ :

$y-y_{0}=f^{\prime}\left(x_{0}\right)\left(x-x_{0}\right)$

$$
\Rightarrow y-y_{0}=\frac{b}{a} \frac{x_{0}}{\sqrt{x_{0}^{2}-a^{2}}}\left(x-x_{0}\right) .
$$

Equação da reta $s$ :

$$
\begin{aligned}
& y-y_{0}=-\frac{1}{f^{\prime}\left(x_{0}\right)}\left(x-x_{0}\right) \\
& \quad \Rightarrow y-y_{0}=-\frac{a}{b} \frac{\sqrt{x_{0}^{2}-a^{2}}}{x_{0}}\left(x-x_{0}\right) .
\end{aligned}
$$

Equação da reta $u$ :

$$
\frac{y-y_{0}}{x-x_{0}}=\frac{y_{0}-0}{x_{0}-c}
$$

$$
\Rightarrow y-y_{0}=\frac{y_{0}}{x_{0}-c}\left(x-x_{0}\right)
$$

Equação da reta $v$ :

$$
\begin{aligned}
\frac{y-y_{0}}{x-x_{0}}=\frac{y_{0}-0}{x_{0}-(-c)} & \Rightarrow \frac{y-y_{0}}{x-x_{0}}=\frac{y_{0}-0}{x_{0}+c} \\
\Rightarrow & y-y_{0}=\frac{y_{0}}{x_{0}+c}\left(x-x_{0}\right) .
\end{aligned}
$$

As coordenados de $Q$ já foram calculadas: 


$$
Q=\left(\frac{a^{2}}{x_{0}}, 0\right) .
$$

Coordenadas do ponto $W$ :

Fazendo $x=0$ na equação da reta $t$ e substituindo $y_{0}=\frac{b}{a} \sqrt{x_{0}^{2}-a^{2}}$, temos:

$$
\begin{aligned}
& y-\frac{b}{a} \sqrt{x_{0}^{2}-a^{2}}=\frac{b}{a} \frac{x_{0}}{\sqrt{x_{0}^{2}-a^{2}}}\left(0-x_{0}\right) \\
& \Rightarrow y=\frac{b}{a} \sqrt{x_{0}^{2}-a^{2}}-\frac{b}{a} \frac{x_{0}^{2}}{\sqrt{x_{0}^{2}-a^{2}}} \\
& \Rightarrow y=\frac{b\left(x_{0}^{2}-a^{2}\right)-b x_{0}^{2}}{a \sqrt{x_{0}^{2}-a^{2}}} \\
& \Rightarrow y=\frac{b x_{0}^{2}-b a^{2}-b x_{0}^{2}}{a \sqrt{x_{0}^{2}-a^{2}}} \\
& \Rightarrow y=\frac{-b a^{2}}{a \sqrt{x_{0}^{2}-a^{2}}} \\
& \Rightarrow y=\frac{-a b}{\sqrt{x_{0}^{2}-a^{2}}} .
\end{aligned}
$$

Logo:

$$
W=\left(0, \frac{-a b}{\sqrt{x_{0}^{2}-a^{2}}}\right) .
$$

Coordenadas do ponto $U$ :

Fazendo $y=0$ na equação da reta $s$ e substituindo $y_{0}=\frac{b}{a} \sqrt{x_{0}^{2}-a^{2}}$, temos:

$$
\begin{aligned}
& 0-\frac{b}{a} \sqrt{x_{0}^{2}-a^{2}}=-\frac{a}{b} \frac{\sqrt{x_{0}^{2}-a^{2}}}{x_{0}}\left(x-x_{0}\right) \\
& \Rightarrow x-x_{0}=\frac{-\frac{b}{a} \sqrt{x_{0}^{2}-a^{2}}}{-\frac{a}{b} \frac{\sqrt{x_{0}^{2}-a^{2}}}{x_{0}}} \\
& \Rightarrow x-x_{0}=\frac{b^{2} x_{0}}{a^{2}} \\
& \Rightarrow x=x_{0}+\frac{b^{2} x_{0}}{a^{2}} \\
& \Rightarrow x=\frac{x_{0}\left(a^{2}+b^{2}\right)}{a^{2}} .
\end{aligned}
$$

Como $a^{2}+b^{2}=c^{2}$, temos que: $x=\frac{c^{2} x_{0}}{a^{2}}$ e ,portanto,

$$
U=\left(\frac{c^{2} x_{0}}{a^{2}}, 0\right) .
$$

Coordenadas do ponto $T$ :

Fazendo $x=0$ na equação da reta $s$ e substituindo $y_{0}=\frac{b}{a} \sqrt{x_{0}^{2}-a^{2}}$, temos:

$$
\begin{aligned}
& y-\frac{b}{a} \sqrt{x_{0}^{2}-a^{2}}=-\frac{a}{b} \frac{\sqrt{x_{0}^{2}-a^{2}}}{x_{0}}\left(0-x_{0}\right) \\
& \Rightarrow y=\frac{b}{a} \sqrt{x_{0}^{2}-a^{2}}+\frac{a \sqrt{x_{0}^{2}-a^{2}}}{b}
\end{aligned}
$$




$$
\begin{aligned}
& \Rightarrow y=\frac{b^{2} \sqrt{x_{0}^{2}-a^{2}}+a^{2} \sqrt{x_{0}^{2}-a^{2}}}{a b} \\
& \Rightarrow y=\frac{\left(a^{2}+b^{2}\right) \sqrt{x_{0}^{2}-a^{2}}}{a b} .
\end{aligned}
$$

Como $a^{2}+b^{2}=c^{2}$, temos que: $y=\frac{c^{2} \sqrt{x_{0}^{2}-a^{2}}}{a b}$.

Logo:

$$
T=\left(0, \frac{c^{2} \sqrt{x_{0}^{2}-a^{2}}}{a b}\right)
$$

Coordenadas do ponto $M$ :

Fazendo $x=0$ na equação da reta $v$, temos que:

$$
\begin{aligned}
& y-y_{0}=\frac{y_{0}}{x_{0}+c}\left(0-x_{0}\right) \\
& \Rightarrow y=y_{0}+\frac{y_{0}}{x_{0}+c}\left(-x_{0}\right) \\
& \Rightarrow y=\frac{x_{0} y_{0}+c y_{0}-x_{0} y_{0}}{x_{0}+c} \\
& \Rightarrow y=\frac{b c \sqrt{x_{0}^{2}-a^{2}}}{a\left(x_{0}+c\right)} .
\end{aligned}
$$

Então:

$$
M=\left(0, \frac{b c \sqrt{x_{0}^{2}-a^{2}}}{a\left(x_{0}+c\right)}\right) .
$$

Agora que já encontramos as coordenadas dos pontos $W, Q, T, U$, e $M$, vamos agora mostrar que o ângulo $\alpha$ é agudo, isto é, mostrar que a semirreta que passa pelos pontos $P$ e $M$ está entre a semirreta que passa pelos pontos $P$ e $T$ e pela semirreta que passa pelos pontos $P$ e $W$. Para isso, vamos mostrar que o ponto $M$ está entre $T$ e $W$. De fato: $\frac{x_{0} \sqrt{a^{2}+b^{2}}+(a-b)^{2}+a b}{x_{0}+c}>0$, pois sendo $a, b, c$ e $x_{0}$ positivos, temos $x_{0} \sqrt{a^{2}+b^{2}}>0,(a-b)^{2} \geq 0, a b>0 \mathrm{e}$ $x_{0}+c>0$. Substituindo $\sqrt{a^{2}+b^{2}}$ por $c$, ficamos com

$$
\begin{aligned}
& \frac{c x_{0}+a^{2}+b^{2}-a b}{x_{0}+c}>0 \\
& \Rightarrow \frac{c x_{0}+c^{2}-a b}{x_{0}+c}>0 \\
& \Rightarrow \frac{c\left(x_{0}+c\right)-a b}{x_{0}+c}>0 \\
& \Rightarrow \frac{c\left(x_{0}+c\right)}{x_{0}+c}-\frac{a b}{x_{0}+c}>0 \\
& \Rightarrow c-\frac{a b}{x_{0}+c}>0 \\
& \Rightarrow \frac{a b}{x_{0}+c}<c .
\end{aligned}
$$

Multiplicando-se ambos os lados da desigualdade acima por $c$, temos que: $\frac{a b c}{x_{0}+c}<c^{2}$.

Dividindo-se ambos os lados da desigualdade acima por $a^{2}$, temos que: 


$$
\begin{aligned}
& \frac{a b c}{a^{2}\left(x_{0}+c\right)}<\frac{c^{2}}{a^{2}} \\
& \Rightarrow \frac{b c}{a\left(x_{0}+c\right)}<\frac{c^{2}}{a^{2}} .
\end{aligned}
$$

Multiplicando-se ambos os lados da desigualdade acima por $\sqrt{x_{0}^{2}-a^{2}}$, temos que:

$$
\frac{b c \sqrt{x_{0}^{2}-a^{2}}}{a\left(x_{0}+c\right)}<\frac{c^{2} \sqrt{x_{0}^{2}-a^{2}}}{a^{2}}
$$

O que mostra que $M$ está abaixo de $T$.

Agora, observe que:

$\left(x_{0}-a\right)\left(x_{0}+a\right)>0$, pois $x_{0}>a$. Logo $x_{0}{ }^{2}-a^{2}>0$ e segue daí que:

$\frac{a^{2}\left(x_{0}+c\right)+c\left(x_{0}^{2}-a^{2}\right)}{a^{2}\left(x_{0}+c\right)}>0$

$\Rightarrow 1+\frac{c\left(x_{0}^{2}-a^{2}\right)}{a^{2}\left(x_{0}+c\right)}>0$

$\Rightarrow \frac{c\left(x_{0}^{2}-a^{2}\right)}{a^{2}\left(x_{0}+c\right)}>-1$.

Como $b>0$, temos que:

$\frac{b c\left(x_{0}^{2}-a^{2}\right)}{a^{2} b\left(x_{0}+c\right)}>-1$.

Multiplicando-se ambos os lados por $a b$, temos que:

$\frac{b c\left(x_{0}^{2}-a^{2}\right)}{a\left(x_{0}+c\right)}>-a b$.

Dividindo-se ambos os lados por $\sqrt{x_{0}^{2}-a^{2}}$, temos que:

$$
\begin{aligned}
& \frac{b c\left(x_{0}{ }^{2}-a^{2}\right)}{a\left(x_{0}+c\right) \sqrt{x_{0}^{2}-a^{2}}}>-\frac{a b}{\sqrt{x_{0}^{2}-a^{2}}} \\
& \Rightarrow \frac{b c\left(x_{0}^{2}-a^{2}\right) \sqrt{x_{0}^{2}-a^{2}}}{a\left(x_{0}+c\right)\left(x_{0}^{2}-a^{2}\right)}>-\frac{a b}{\sqrt{x_{0}^{2}-a^{2}}} \\
& \Rightarrow \frac{b c \sqrt{x_{0}^{2}-a^{2}}}{a\left(x_{0}+c\right)}>-\frac{a b}{\sqrt{x_{0}^{2}-a^{2}}} . \\
& \text { O que mostra que } M \text { está acima de } W .
\end{aligned}
$$

Resumindo:

$$
-\frac{a b}{\sqrt{x_{0}^{2}-a^{2}}}<\frac{b c \sqrt{x_{0}^{2}-a^{2}}}{a\left(x_{0}+c\right)}<\frac{c^{2} \sqrt{x_{0}^{2}-a^{2}}}{a^{2}} .
$$

Então, o ponto $M$ está entre $T$ e $W$ como queríamos demostrar, e portanto a semirreta que passa pelos pontos $P$ e $M$ está entre a semirreta que passa pelos pontos $P$ e $T$ e pela semirreta que passa pelos pontos $P$ e $W$. Com isso, podemos concluir que o ângulo $\alpha$ é agudo.

Vamos agora mostrar que o ângulo $\beta$ é agudo. Para isso, basta mostrar que a semi reta que passa pelos pontos $P$ e $F_{1}$ está entre a semi reta que passa pelos pontos $P$ e $Q$ e pela semi reta que passa pelos pontos $P$ e $U$. Equivalentemente, temos que mostrar que o ponto $F_{1}$ está entre $Q$ e $U$. 
Lembramos que $0<a<c$ e que $0<a<x_{0}$. Assim, vale que $a^{2}<c x_{0}$. Com isso segue-se primeiro que $\frac{a^{2}}{x_{0}}<c$. Multiplicando-se $a^{2}<c x_{0}$ dos dois lados por $c$, que é positivo, nos leva a $c a^{2}<c^{2} x_{0}$, ou ainda $c<\frac{c^{2} x_{0}}{a^{2}}$. Resumindo:

$$
\frac{a^{2}}{x_{0}}<c<\frac{c^{2} x_{0}}{a^{2}} .
$$

Vemos então que o ponto $F_{1}$ está entre $Q$ e $U$, e portanto a semirreta que passa pelos pontos $P$ e $F_{1}$ está entre a semirreta que passa pelos pontos $P$ e $Q$ e pela semirreta que passa pelos pontos $P$ e $U$. Com isso, podemos concluir que o ângulo $\beta$ é agudo.

Agora que já mostramos que $\alpha$ e $\beta$ são agudos, iremos demonstrar que $\operatorname{tg} \alpha=\operatorname{tg} \beta$ e concluir que $\alpha=\beta$.

A demonstração a seguir envolve contas com o coeficiente angular da reta que passa por $P$ e $F_{1}$, mas se $x_{0}=c$ a essa reta é vertical, por isso vamos supor no momento $x_{0} \neq c$. Começamos com as seguintes considerações:

$m_{1}$ : coeficiente angular da reta que passa pelos pontos $F_{2}=(-c, 0)$ e $P=\left(x_{0}, y_{0}\right)$.

$$
m_{1}=\frac{y_{0}-0}{x_{0}-(-c)} \Rightarrow m_{1}=\frac{y_{0}}{x_{0}+c}
$$

$m_{2}$ : coeficiente angular da reta $t$ tangente em $x_{0}$.

$$
m_{2}=f^{\prime}\left(x_{0}\right) \Rightarrow m_{2}=\frac{b}{a} \frac{x_{0}}{\sqrt{x_{0}^{2}-a^{2}}} .
$$

Observando que o ponto $P=\left(x_{0}, y_{0}\right)$ pertence à hipérbole, temos que:

$$
y_{0}=\frac{b}{a} \sqrt{x_{0}^{2}-a^{2}}
$$

ou ainda

$$
\sqrt{x_{0}^{2}-a^{2}}=\frac{a y_{0}}{b}
$$

Com isso,

$$
m_{2}=\frac{b}{a} \frac{x_{0}}{\left(a y_{0} / b\right)}=\frac{b^{2} x_{0}}{a^{2} y_{0}} .
$$

$m_{3}$ : coeficiente angular da reta que passa pelos pontos $F_{1}=(c, 0)$ e $P=\left(x_{0}, y_{0}\right)$.

$$
m_{3}=\frac{y_{0}-0}{x_{0}-c} \Rightarrow m_{3}=\frac{y_{0}}{x_{0}-c} .
$$


Observe a figura a seguir

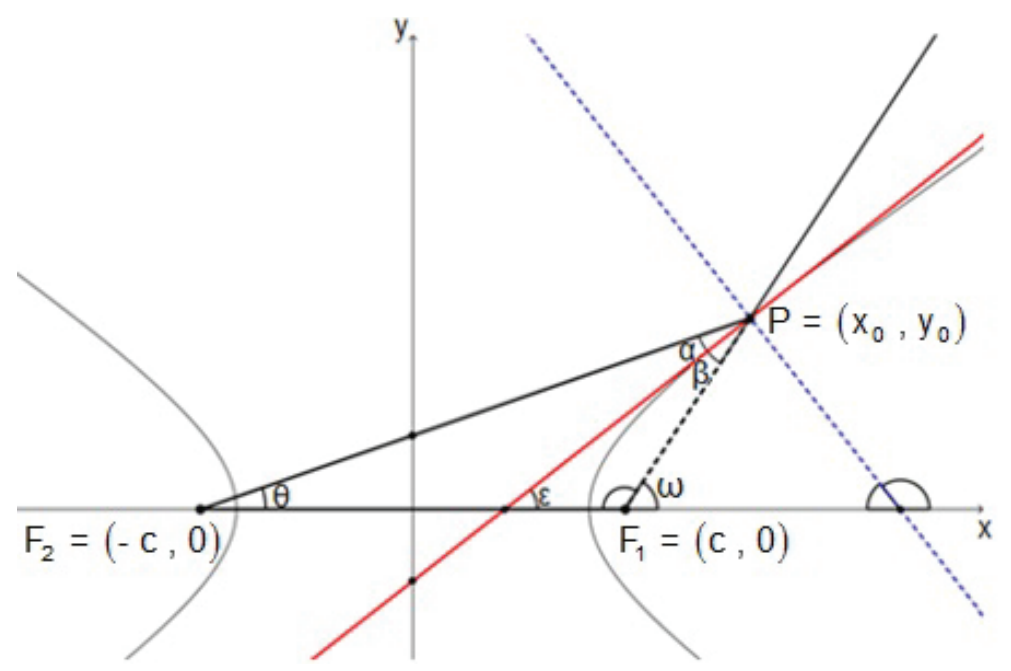

Figura 4.5: Hipérbole Reta Normal

Podemos concluir que:

$$
\begin{aligned}
& m_{1}=\operatorname{tg} \theta \\
& m_{2}=f^{\prime}\left(x_{0}\right)=\operatorname{tg} \varepsilon \\
& m_{3}=\operatorname{tg} \omega .
\end{aligned}
$$

Além disso:

$$
\begin{aligned}
& \varepsilon=\alpha+\theta \Rightarrow \alpha=\varepsilon-\theta, \\
& \Rightarrow \omega=\beta+\varepsilon \Rightarrow \beta=\omega-\varepsilon .
\end{aligned}
$$

Logo:

$$
\begin{aligned}
& \operatorname{tg} \alpha=\operatorname{tg}(\varepsilon-\theta)=\frac{\operatorname{tg} \varepsilon-\operatorname{tg} \theta}{1+\operatorname{tg} \varepsilon \operatorname{tg} \theta}=\frac{m_{2}-m_{1}}{1+m_{2} m_{1}} \\
& \Rightarrow \operatorname{tg} \alpha=\frac{\frac{b^{2} x_{0}}{a^{2} y_{0}}-\frac{y_{0}}{x_{0}+c}}{1+\frac{b^{2} x_{0}}{a^{2} y_{0}} \cdot \frac{y_{0}}{x_{0}+c}}=\frac{\frac{b^{2} x_{0}\left(x_{0}+c\right)-a^{2} y_{0}}{a^{2} y_{0}\left(x_{0}+c\right)}}{\frac{a^{2} y_{0}\left(x_{0}+c\right)+b^{2} x_{0} y_{0}}{a^{2} y_{0}\left(x_{0}+c\right)}} \\
& \Rightarrow \operatorname{tg} \alpha=\frac{b^{2} x_{0}^{2}+b^{2} c x_{0}-a^{2} y_{0}^{2}}{a^{2} x_{0} y_{0}+a^{2} c y_{0}+b^{2} x_{0} y_{0}} .
\end{aligned}
$$

Lembrando que $y_{0}=\frac{b}{a} \sqrt{x_{0}^{2}-a^{2}}$, podemos substituir $a^{2} y_{0}^{2}$ por $b^{2}\left(x_{0}^{2}-\right.$ $\left.a^{2}\right)$ e obtemos

$$
\begin{aligned}
& \operatorname{tg} \alpha=\frac{b^{2} x_{0}{ }^{2}+b^{2} c x_{0}-b^{2}\left(x_{0}{ }^{2}-a^{2}\right)}{a^{2} x_{0} y_{0}+a^{2} c y_{0}+b^{2} x_{0} y_{0}} \\
& \Rightarrow \operatorname{tg} \alpha=\frac{b^{2} x_{0}{ }^{2}+b^{2} c x_{0}-b^{2} x_{0}{ }^{2}+a^{2} b^{2}}{a^{2} x_{0} y_{0}+a^{2} c y_{0}+b^{2} x_{0} y_{0}} .
\end{aligned}
$$

Colocando $x_{0} y_{0}$ em evidência no denominador, temos $\operatorname{tg} \alpha=\frac{b^{2} x_{0}{ }^{2}+b^{2} c x_{0}-b^{2} x_{0}{ }^{2}+a^{2} b^{2}}{x_{0} y_{0}\left(a^{2}+b^{2}\right)+a^{2} c y_{0}}$.

Lembrando que $a^{2}+b^{2}=c^{2}$, obtemos agora 
$\operatorname{tg} \alpha=\frac{b^{2} c x_{0}+a^{2} b^{2}}{c^{2} x_{0} y_{0}+a^{2} c y_{0}}$
$\Rightarrow \operatorname{tg} \alpha=\frac{b^{2}\left(c x_{0}+a^{2}\right)}{c y_{0}\left(c x_{0}+a^{2}\right)}$.

Finalmente,

$$
\operatorname{tg} \alpha=\frac{b^{2}}{c y_{0}}
$$

E também,

$$
\begin{aligned}
& \operatorname{tg} \beta=\operatorname{tg}(\omega-\varepsilon)=\frac{\operatorname{tg} \omega-\operatorname{tg} \varepsilon}{1+\operatorname{tg} \omega \operatorname{tg} \varepsilon}=\frac{m_{3}-m_{2}}{1+m_{3} m_{2}} \\
& \Rightarrow \operatorname{tg} \beta=\frac{\frac{y_{0}}{x_{0}-c}-\frac{b^{2} x_{0}}{a^{2} y_{0}}}{1+\frac{y_{0}}{x_{0}-c} \cdot \frac{b^{2} x_{0}}{a^{2} y_{0}}}=\frac{\frac{a^{2} y_{0}^{2}-b^{2} x_{0}\left(x_{0}-c\right)}{a^{2} y_{0}\left(x_{0}-c\right)}}{\frac{a^{2} y_{0}\left(x_{0}-c\right)+b^{2} x_{0} y_{0}}{a^{2} y_{0}\left(x_{0}-c\right)}} \\
& \Rightarrow \operatorname{tg} \beta=\frac{a^{2} y_{0}{ }^{2}-b^{2} x_{0}{ }^{2}+b^{2} c x_{0}}{a^{2} x_{0} y_{0}-a^{2} c y_{0}+b^{2} x_{0} y_{0}} .
\end{aligned}
$$

Substituindo $a^{2} y_{0}^{2}$ por $b^{2}\left(x_{0}^{2}-a^{2}\right)$, obtemos

$$
\begin{aligned}
& \operatorname{tg} \beta=\frac{b^{2}\left(x_{0}{ }^{2}-a^{2}\right)-b^{2} x_{0}{ }^{2}+b^{2} c x_{0}}{a^{2} x_{0} y_{0}-a^{2} c y_{0}+b^{2} x_{0} y_{0}} \\
& \Rightarrow \operatorname{tg} \beta=\frac{b^{2} x_{0}{ }^{2}-b^{2} a^{2}-b^{2} x_{0}{ }^{2}+b^{2} c x_{0}}{a^{2} x_{0} y_{0}-a^{2} c y_{0}+b^{2} x_{0} y_{0}} .
\end{aligned}
$$

Colocando $x_{0} y_{0}$ em evidência no denominador, temos

$\operatorname{tg} \beta=\frac{b^{2} x_{0}{ }^{2}-b^{2} a^{2}-b^{2} x_{0}^{2}+b^{2} c x_{0}}{x_{0} y_{0}\left(a^{2}+b^{2}\right)-a^{2} c y_{0}}$

$\Rightarrow \operatorname{tg} \beta=\frac{b^{2} c x_{0}-a^{2} b^{2}}{x_{0} y_{0}\left(a^{2}+b^{2}\right)-a^{2} c y_{0}}$.

Com $a^{2}+b^{2}=c^{2}$,

$\operatorname{tg} \beta=\frac{b^{2} c x_{0}-a^{2} b^{2}}{c^{2} x_{0} y_{0}-a^{2} c y_{0}}$

$\Rightarrow \operatorname{tg} \beta=\frac{b^{2}\left(c x_{0}-a^{2}\right)}{c y_{0}\left(c x_{0}-a^{2}\right)}$.

Daí

$$
\operatorname{tg} \beta=\frac{b^{2}}{c y_{0}}
$$

Com isso, concluímos que $\operatorname{tg} \alpha=\operatorname{tg} \beta$ no caso em que $x_{0} \neq c$ e $x_{0} \neq a$.

Vejamos o caso $x_{0}=c$, ou seja, caso $P=\left(c, \frac{b}{a} \sqrt{c^{2}-a^{2}}\right)=\left(c, \frac{b^{2}}{a}\right)$.

As contas feitas acima pata $\operatorname{tg} \alpha$ continuam valendo, ou seja, $\operatorname{tg} \alpha=\frac{b^{2}}{c y_{0}}$.

Substituindo $y_{0}$ por $\frac{b^{2}}{a}$, temos $\operatorname{tg} \alpha=\frac{b^{2}}{c b^{2} / a}=\frac{1}{c / a}=\frac{a}{c}$.

Agora para calcular tg $\beta$ lembramos que $Q F_{1} P$ é retângulo:

$$
\operatorname{tg} \beta=\frac{\operatorname{dist}\left(Q, F_{1}\right)}{\operatorname{dist}\left(F_{1}, P\right)}=\frac{c-\frac{a^{2}}{c}}{\frac{b^{2}}{a}}=\frac{c^{2}-a^{2}}{c} \cdot \frac{a}{b^{2}}=\frac{a}{c} \text {. }
$$




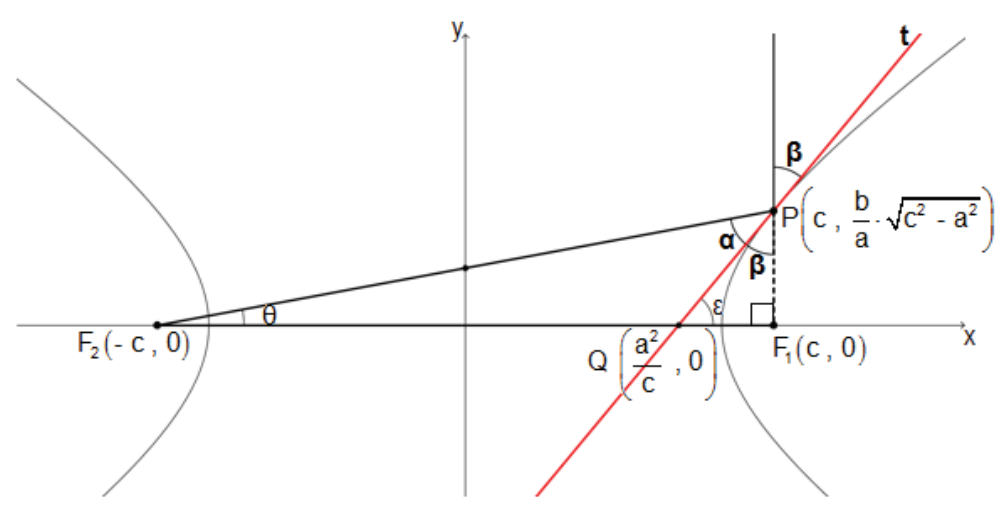

Figura 4.6: Hipérbole ângulo reto

Concluímos assim que $\operatorname{tg} \alpha=\operatorname{tg} \beta$ também no caso em que $x_{0}=c$. Como $\alpha$ e $\beta$ são ângulos agudos segue que:

$$
\alpha=\beta .
$$

Resta apenas discutirmos o caso em que $x_{0}=a$, ou seja, $P=(a, 0)$. Nesse caso a propriedade reflexiva é satisfeita trivialmente, pois a reta por $P$ e $F_{1}$ e a reta por $P$ e $F_{2}$ são ambas iguais ao eixo $x$ e a reta tangemte à elipse em $P$ é vertical de equação $x=a$.

Com isso, fica demonstrada a propriedade reflexiva da hipérbole. 


\section{5}

\section{Aplicações}

Podemos perceber que a parábola tem propriedades reflexivas interessantes. Uma aplicação desta propriedade pode ser observada na construção das antenas parabólicas, considerando raios de luz paralelos ao eixo da parábola e sendo refletidos para o foco, onde, por exemplo, é colocado um receptor de sinal para receber os sinais via satélite (figura 5.1).
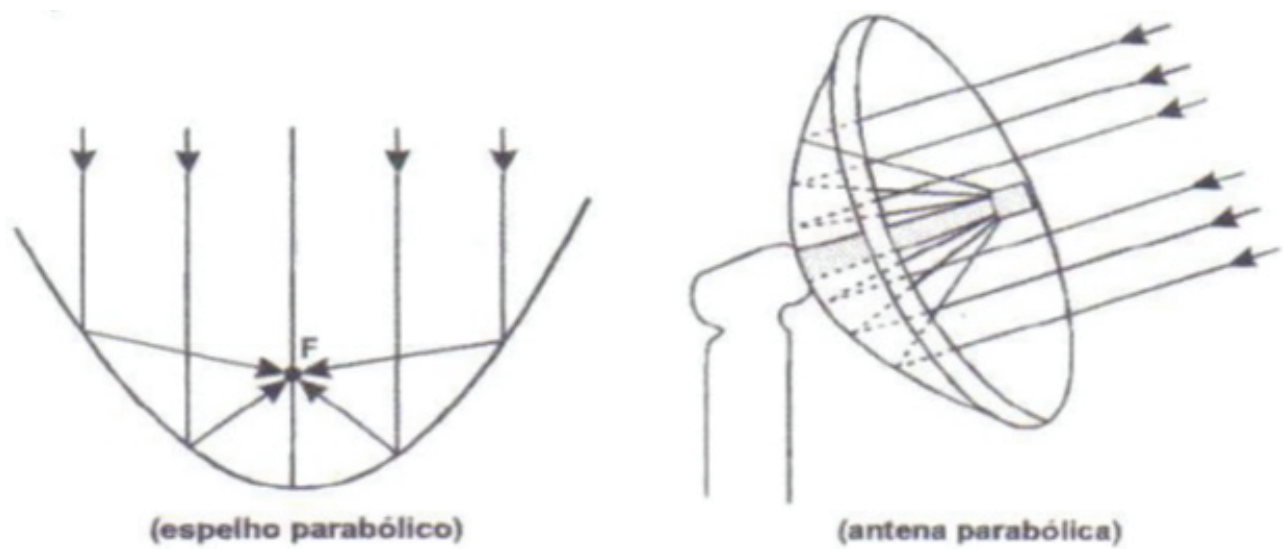

Figura 5.1: Antena Parabólica

Outra aplicação interessante é observada na construção dos faróis de automóveis, onde a lâmpada é localizada no foco dessa parábola em que os raios de luz são incididos e refletidos na superfície parabólica (figura 5.2).
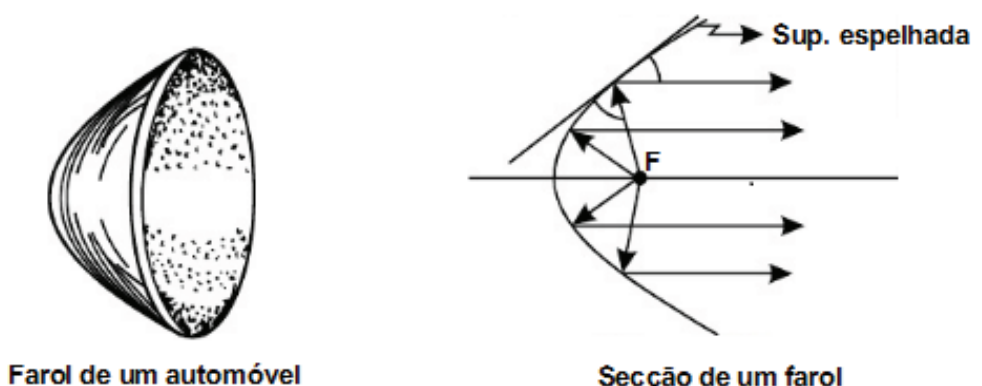

Figura 5.2: Farol de Carro

Nos dois casos acima as superfícies são parabólicas e isso faz com que os sinais de rádio e luz sejam amplificados para o foco da parábola. 
Uma outra aplicação com superfícies parabólicas pode ser observada no fogão solar onde se coloca um suporte de forma que a panela esteja no foco da parábola como nas figuras 5.3 e 5.4 .

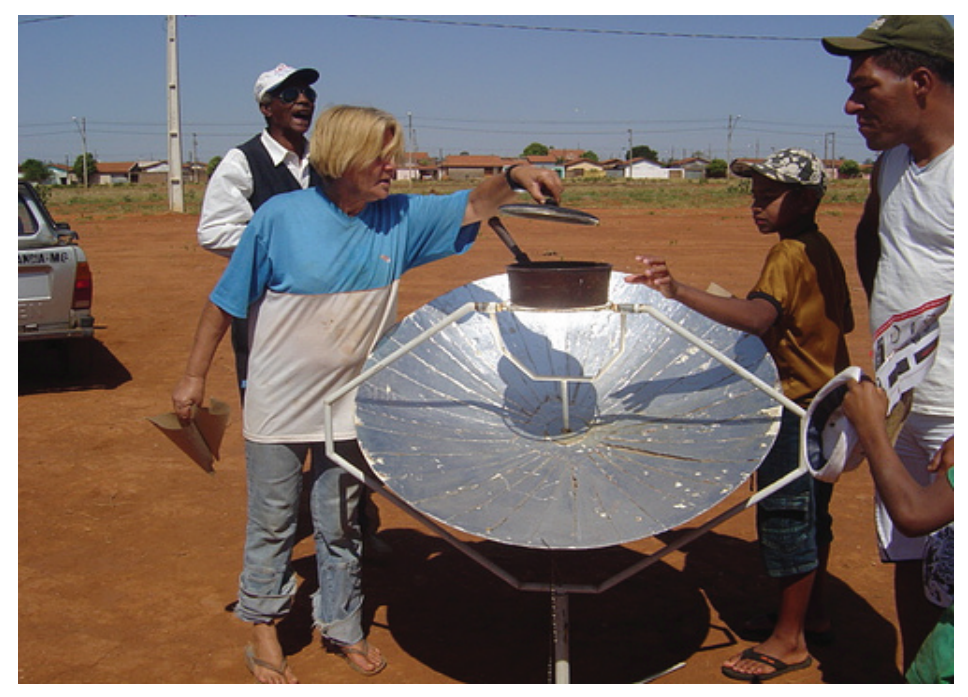

Figura 5.3: Fogão Solar 1

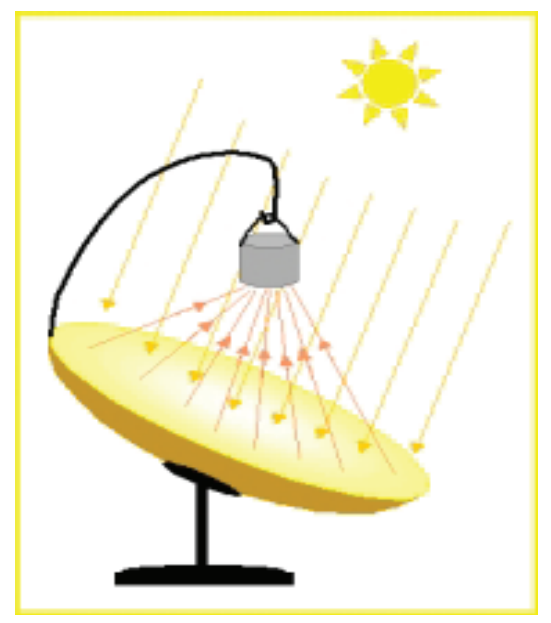

Figura 5.4: Fogão Solar 2

É fácil ver que não funcionaria trocar um parabolóide por uma esfera, mas poderíamos nos perguntar se uma outra superfície côncava espelhada poderia ser usada no lugar da parábola, ou seja, se outra forma de espelho concentraria raios paralelos refletidos em um foco. Em (Dutenhefner) encontramos uma demonstração usando cálculo diferencial a uma variável de que de fato a parábola é a única curva com tal propriedade.

Já com relação à elipse, podemos observar sua propriedade reflexiva nos refletores odontológicos de maneira que o grande objetivo desses aparelhos 
é concentrar o máximo de luz, sem que essa luz cause desconforto para o tratamento dentário do paciente.

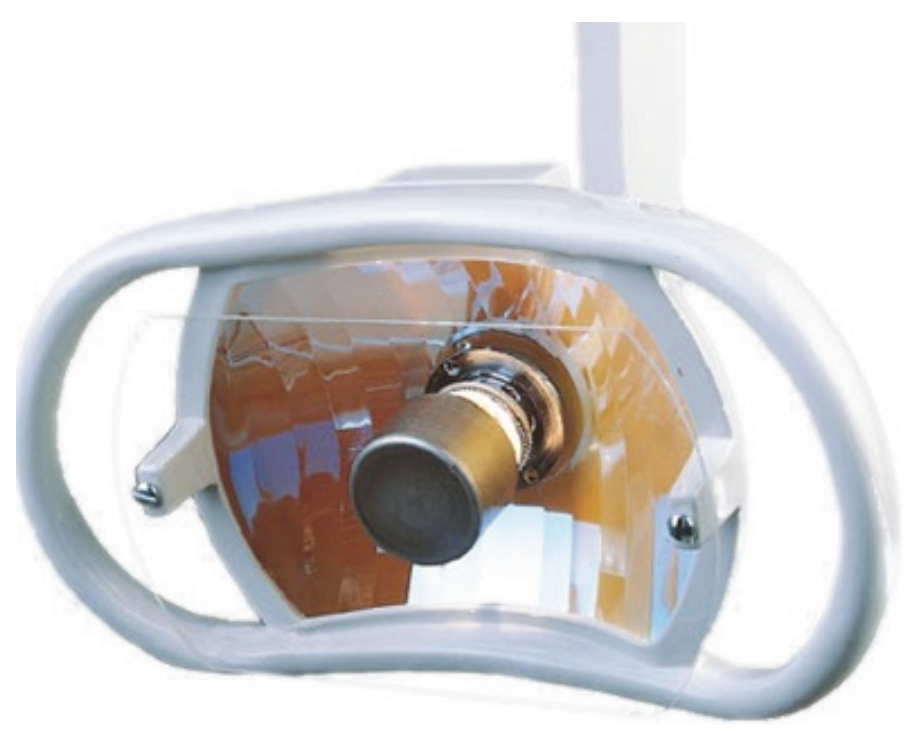

Figura 5.5: Refletor Odontológico 1

Observe na figura 5.6, o funcionamento do refletor odontológico. A luz da lâmpada é concentrada através do espelho no outro foco, que é ajustado pelo dentista para estar num ponto dentro da boca de seu paciente.
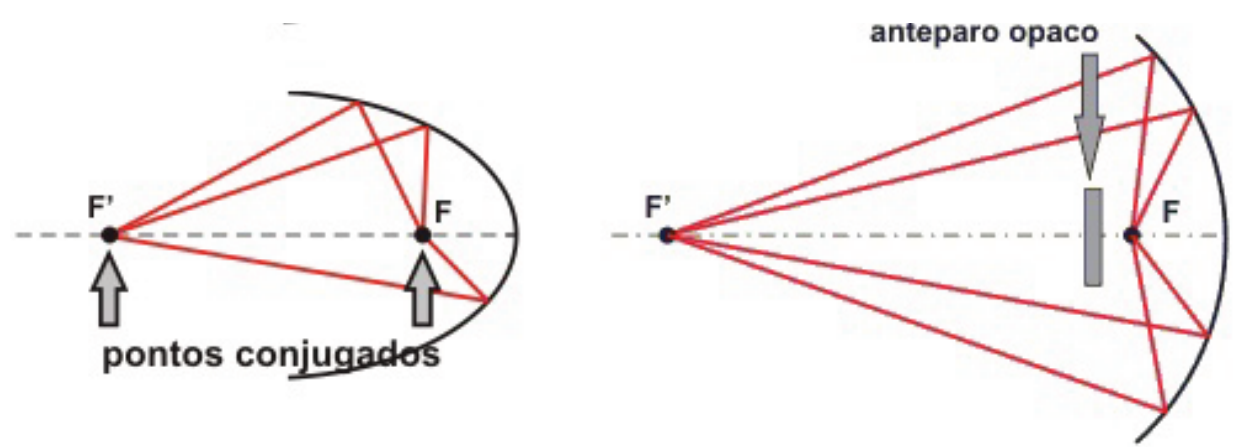

Figura 5.6: Refletor Odontológico 2

Também podemos observar a presença de aparelhos em formato de elipse em tratamentos de radioterapia, onde os raios são incididos na superfície elíptica e refletidos no tecido doente, não afetando outros tecidos na vizinhança desse tecido doente.

Finalmente, a hipérbole possui sua propriedade reflexiva na construção de telescópios de reflexão. Esses telescópios funcionam da seguinte maneira: O espelho maior é parabólico e o menor é hiperbólico e tais espelhos são construídos de maneira que seus eixos coincidam e que um dos focos do espelho hiperbólico seja o foco do espelho parabólico. 
Os raios de luz incidem sobre o espelho parabólico e refletem para o foco desse espelho. Como também este é o foco da hipérbole, os raios de luz se refletem e se dirigem em direção ao outro foco. Estes raios de luz passam através de uma fenda no centro do espelho parabólico no qual está uma lente ocular que permite corrigir a trajetória da luz, que chega finalmente aos olhos do observador.

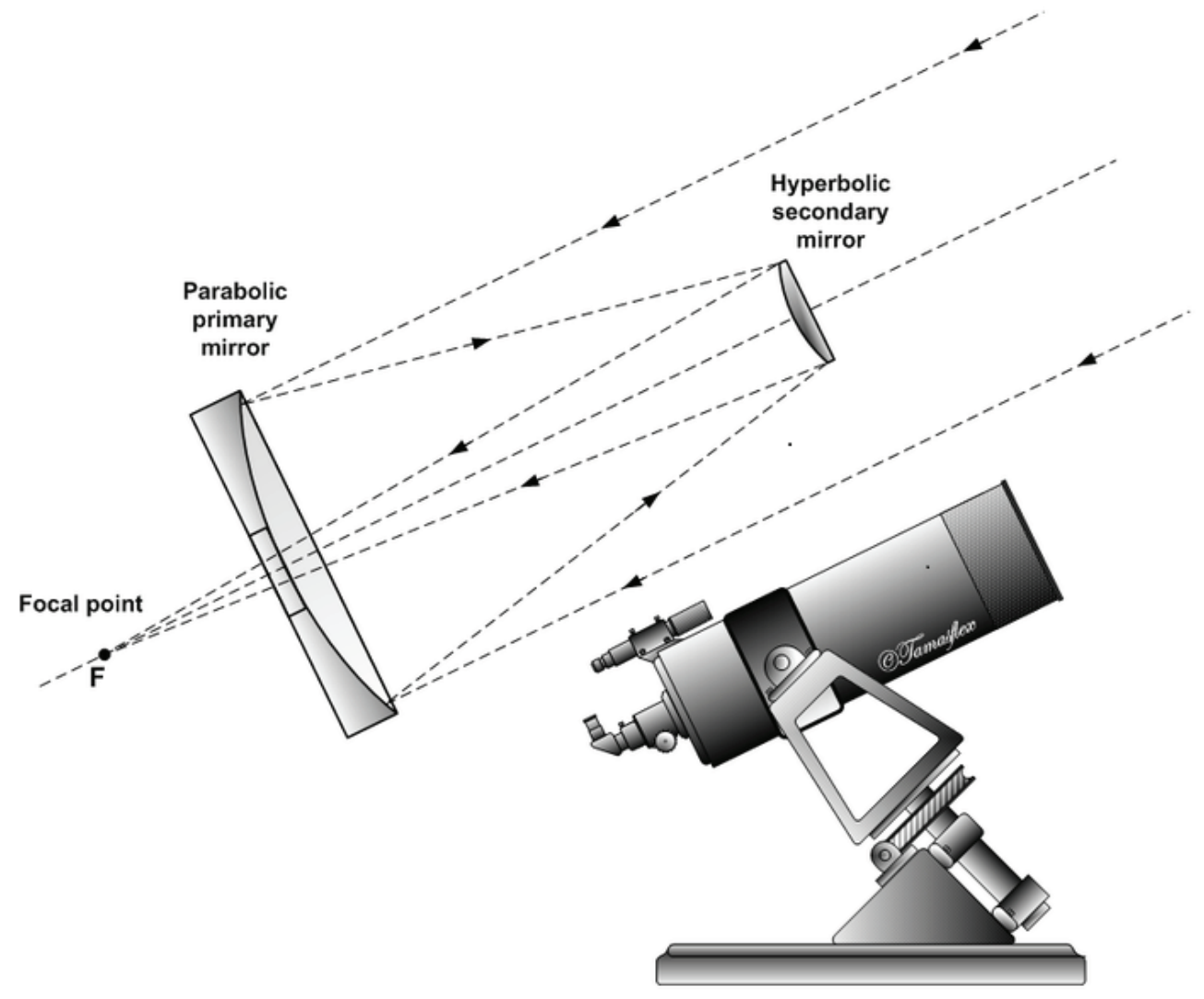

Figura 5.7: Telescópio

Esse telescópio é feito com combinação de curvas. Outros exemplos de combinação de curvas e propriedades reflexivas podem ser encontrados em (Downs). 


\section{Referências das Fotos e Figuras do Capítulo:}

(1) http://www.sempresustentavel.com.br/solar.htm (fogão solar)

(2) http://tupidataba.blogspot.com.br/2005/07/fogo-solar-feito-comembalagem-longa.html (fogão solar)

(3) http://en.wikipedia.org/wiki/Cassegrain_reflector (telescópio)

(4) http://www.ebah.com.br/content/ABAAAfxCEAG/quadricas-marlon (farol de carro)

(5) http://alfaconnection.net/pag_avsf/luz0303.htm (aparelho odontológico)

(6) http://www.kaeleodonto.com.br/ (aparelho odontológico) 


\section{6 \\ Projeto de Ensino}

Esse trabalho pode ser abordado como tema de uma aula a ser aplicada no ensino médio. Sabemos que os alunos têm uma grande dificuldade em entender a associação entre uma curva e uma equação, ou seja, entre geometria e álgebra.

Um exemplo clássico de sala de aula é quando o professor do $1^{\circ}$ ano do ensino médio, ao introduzir o gráfico de uma função quadrática, afirma que a equação $y=a x^{2}+b x+c$ representa uma parábola, mas isso sem definir parábola como lugar geométrico e nem como secção cônica. A professora Renata, orientadora desse TCC, vem trabalhado há anos com alunos da disciplina de cálculo de primeiro semestre na PUC-Rio e relatou que a maioria dos alunos chega à universidade sem saber o que é uma parábola, sabem que tem um formato parecido com um "U", mas alguns acham que se a figura estiver inclinada (diretriz não horizontal) a figura deixa de ser uma parábola. Também não é rara a ausência da definição nos livros didáticos usados nas escolas.

O uso de novas tecnologias na escola pode alavancar a aprendizagem dos alunos e estabelecer ligações com o que o aluno já conhece com o que é novo. Tendo infraestrutura, podemos incluir atividades fazendo o uso do GeoGebra, levando os alunos a raciocinar sobre cônicas e suas respectivas representações cartesianas. Uma grande vantagem de se utilizar um programa como o GeoGebra é o poder de movimentação de objetos, proporcionando uma boa visualização sobre o problema que estamos tratando.

Na escola onde leciono, Pedro II, iremos realizar um projeto de aprofundamento, onde iremos abordar conteúdos matemáticos para alunos que pretendem seguir a área tecnológica ou científica ou que simplesmente tenham interesse em matemática. A seguir mostraremos um esboço de um roteiro para aulas sobre parábola com caráter interdisciplinar, onde usaremos a propriedade reflexiva como motivação e contextualização. 


\section{Roteiro:}

1. Definir parábola como lugar geométrico.

2. Desenhar parte de uma parábola com régua, esquadro e uma corda:

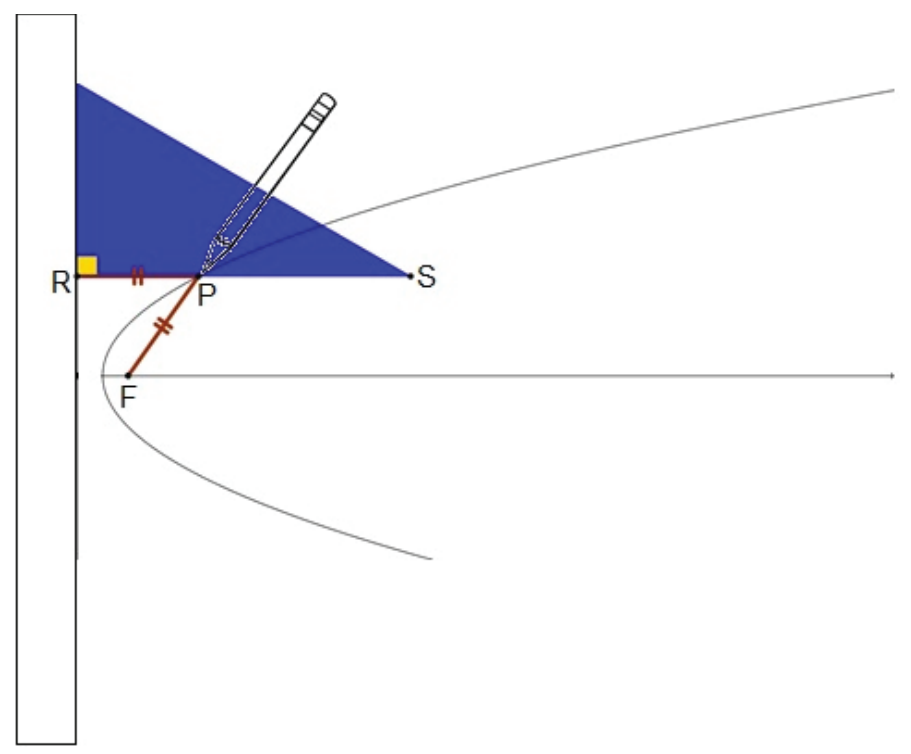

Figura 6.1: Construção de Parábola

3. Mencionar antena parabólica e perguntar se os alunos imaginam a relação com parábola.

4. Deduzir a equação no caso foco $F=(0,1 / 4)$ e diretriz de equação $y=-1 / 4$ : Usar apenas o Teorema de Pitágoras para encontrar $\operatorname{dist}(P, F)$ sem falar de fórmula de distância e calcular a distância entre $P$ e a diretriz visualmente.

5. Avisar que as equações que são estudadas na escola sempre terão a reta diretriz horizontal.

6. Discutir o gráfico de equação $y=-x^{2}$ e concavidade.

7. Usar o GeoGebra para relacionar gráficos e equações $y=a x^{2}+b x+c$.

8. Discutir reflexão fazendo perguntas como, por exemplo, se uma bola de sinuca bater na lateral da mesa como eles acham que será a trajetória, ou se uma luz bater em uma superfície espelhada como eles acham que a luz será refletida, etc.

9. Procurar com os alunos figuras de antenas parabólicas na Internet e afirmar que de fato existe a relação com a parábola. 
10. Explicar a propriedade reflexiva da parábola, sem demonstrar. Aplicar para a antena.

11. Usar o GeoGebra novamente para ilustrar a propriedade reflexiva.

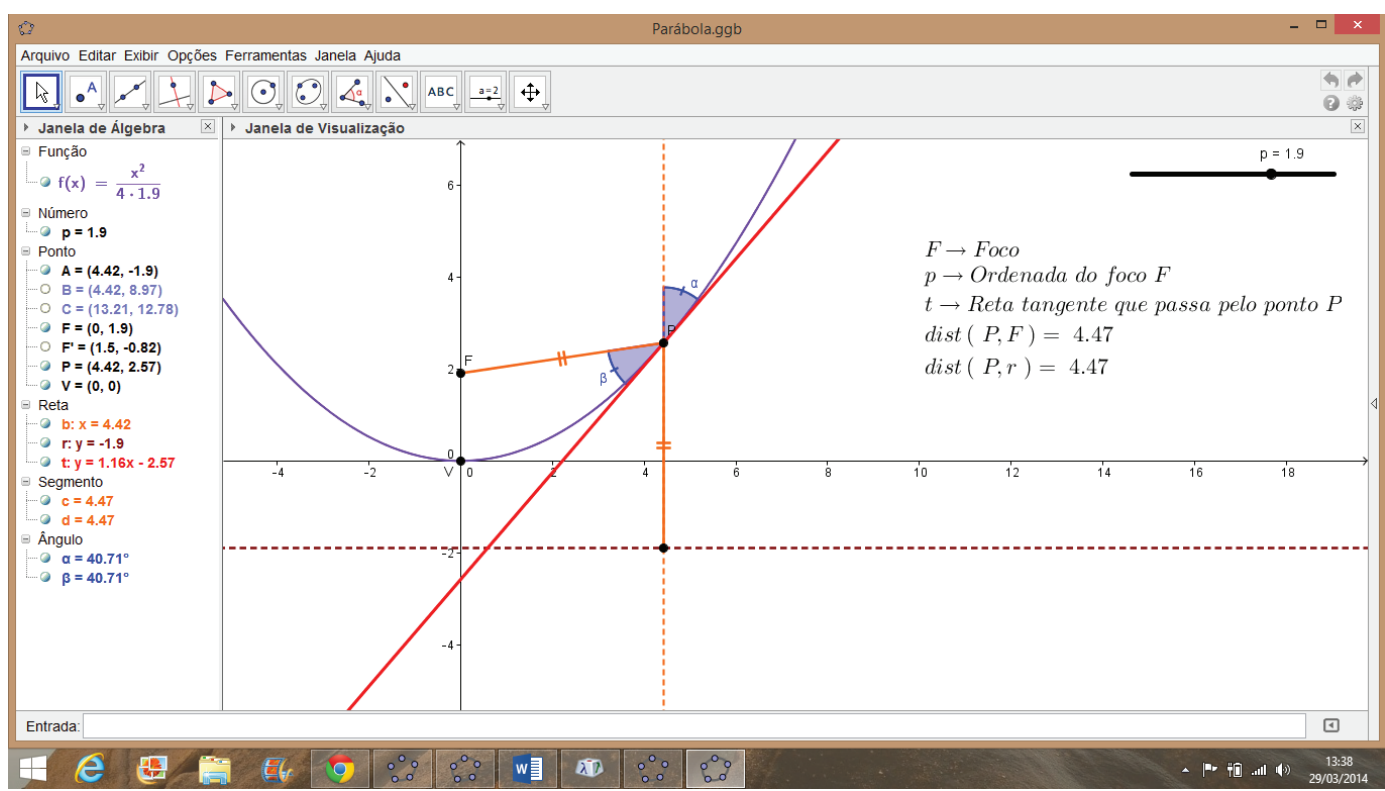

Figura 6.2: Uso do GeoGebra

12. Mostrar aos alunos que outras curvas não possuem as propriedades reflexivas da parábola usando como ferramenta o link: geogebratube.org/studenta/m100696.

13. Procurar com os alunos vídeos na Internet com mais aplicações como, por exemplo, a reportagem feita sobre o fogão solar no sertão da Paraíba: https://www.youtube.com/watch?v=j5zddIut_9g.

14. Relacionar com a disciplina de física.

Gostaria de fazer uma observação final sobre o uso de Internet. É bastante válido o uso de computador, celular, tablets. A pesquisa na Internet pode ser extremamente rica. Porém deve haver um crivo, uma espécie de filtro de pesquisa, e o papel do professor é de orientar os alunos de forma adequada sobre o que é válido ser utilizado como fonte de pesquisa ou não. Só para citar um exemplo, não é raro encontrarmos desenhos de parábolas que na verdade são semicircunferências. 


\section{Conclusão e trabalhos futuros}

Este trabalho foi focado na apresentação e demonstração das propriedades reflexivas das cônicas. Uma aplicação importante deste trabalho é o fato de observar essas propriedades reflexivas no nosso cotidiano, como observamos no capítulo 5 . Tais aplicações são importantes, pois elas, de alguma maneira, tem a intenção de atrair, convidar e levar os alunos a estudarem tais propriedades, juntamente com o professor que tem o papel importante de orientar esses alunos a concluírem que cada cônica possui propriedades reflexivas interessantes que influenciam no nosso cotidiano.

Planejamos continuar este trabalho como foi observado no capítulo 6 . Primeiramente, temos a intenção de apresentar as propriedades reflexivas, fazendo o uso de vídeos e apresentações interativas usando o software GeoGebra e posteriormente desenvolver e demonstrar todas as propriedades com os alunos, e é nesse contexto que se insere o Profmat, pois é importante que o professor seja bem preparado, que tenha os conceitos bem estabelecidos e organizados para que ele possa promover e planejar boas aulas. A proposta do profmat consiste em aprender para ensinar.

Para finalizar, deixo aqui neste trabalho um projeto de ensino, onde podemos nos basear nele para planejar um plano de curso interessante, fazendo com que o aluno se sinta confortável e capaz de realizar tarefas que permitam a ele, a concluir e deduzir todas as propriedades reflexivas. É importante deixar registrado que nossa intenção, com esse projeto de ensino, é primeiramente aplicar, relatar e verificar através de experiências em sala de aula, o que podemos concluir e, após essas experiências, podemos propor um plano de aulas para podermos ministrá-las. Ao leitor deste trabalho, deixo aqui o meu contato via email lsouzagoncalves.mat@gmail.com, onde podemos entrar em contato para podermos trocar experiências que possam contribuir para o melhor desenvolvimento deste projeto de ensino. 


\section{Referências Bibliográficas}

[Avila] ÁVILA, G.. A hipérbole e os telescópios. Revista do professor de matemática, (34):22-27, 1997.

[Downs] DOWNS, J. W.. Practical Conic Sections. Dover Publications, Palo Alto, CA, 1993. 5

[Dutenhefner] DUTENHEFNER, F.. Caracterização das seções cônicas através das suas propriedades . UFMG, MG. 5

[Oliveira] OLIVEIRA, O.. Cônicas (Propriedades de Reflexão). USP, São Paulo. 1

[Sato] SATO, J.. As Cônicas e suas aplicações. UFU, MG. 1

[Stewart] STEWART, J.. Cálculo. Pioneira, São Paulo, 2001.

[Wagner] WAGNER, E.. Porque as antenas são parabólicas. Revista do professor de matemática, (33):10-15, 1997. 
A

\section{Apêndice com Recíproca da Eq. da Elipse}

Seja $P=(x, y)$ um ponto do plano tal que $\frac{x^{2}}{a^{2}}+\frac{y^{2}}{b^{2}}=1$ para $a>b>0$. Vamos provar que $P$ pertence à elipse de focos $F_{1}=(c, 0) \mathrm{e}$ $F_{2}=(-c, 0)$ e constante $2 a$, onde $c=\sqrt{a^{2}-b^{2}}$, ou seja, vamos provar que $\operatorname{dist}\left(P, F_{1}\right)+\operatorname{dist}\left(P, F_{2}\right)=2 a$.

Vamos fazer algumas observações sobre os pontos que estamos considerando antes de começarmos as contas. Como $a>b$, temos $c>0$, e portanto $F_{1} \neq F_{2}$. Além disso, $F_{1} \neq P$ e $F_{2} \neq P$, pois as coordenadas de $F_{1}$ e de $F_{2}$ não satisfazem a equação $\frac{x^{2}}{a^{2}}+\frac{y^{2}}{b^{2}}=1$. De fato, colocando as coordenadas de $F_{1}$ ou de $F_{2}$ na equação, teríamos $\frac{c^{2}}{a^{2}}+\frac{0^{2}}{b^{2}}=1$, ou seja, $a^{2}=c^{2}$, o que não pode acontecer uma vez que $a^{2}=c^{2}+b^{2}$ e $b>0$.

Substituindo $b^{2}$ por $a^{2}-c^{2}$ na equação $\frac{x^{2}}{a^{2}}+\frac{y^{2}}{b^{2}}=1$, ficamos com:

$$
\frac{x^{2}}{a^{2}}+\frac{y^{2}}{a^{2}-c^{2}}=1
$$

Colocando as frações com o mesmo denominador, obtemos

$\frac{x^{2}\left(a^{2}-c^{2}\right)}{a^{2}\left(a^{2}-c^{2}\right)}+\frac{a^{2} y^{2}}{a^{2}\left(a^{2}-c^{2}\right)}=1$.

Multiplicando a equação por $a^{2}\left(a^{2}-c^{2}\right)$, chegamos em

$$
\begin{aligned}
& x^{2}\left(a^{2}-c^{2}\right)+a^{2} y^{2}=a^{2}\left(a^{2}-c^{2}\right) \\
& \Rightarrow a^{2} x^{2}-c^{2} x^{2}+a^{2} y^{2}=a^{4}-a^{2} c^{2} \\
& \Rightarrow a^{2} x^{2}+a^{2} c^{2}+a^{2} y^{2}=a^{4}+c^{2} x^{2} .
\end{aligned}
$$

Somando $2 a^{2} c x$ nos dois lados,

$$
\begin{aligned}
& a^{2} x^{2}+2 a^{2} c x+a^{2} c^{2}+a^{2} y^{2}=a^{4}+2 a^{2} c x+c^{2} x^{2} \\
& \Rightarrow a^{2}\left(x^{2}+2 c x+c^{2}+y^{2}\right)=a^{4}+2 a^{2}(c x)+(c x)^{2} \\
& \Rightarrow a^{2}\left((x+c)^{2}+y^{2}\right)=\left(a^{2}+x c\right)^{2} .
\end{aligned}
$$

Agora temos duas possibilidades:

$$
a^{2}+x c=a \sqrt{(x+c)^{2}+y^{2}}
$$

$\mathrm{ou}$

$$
a^{2}+x c=-a \sqrt{(x+c)^{2}+y^{2}}
$$

Vamos trabalhar primeiro com a Eq. 1. Multiplicando a equação por 4, temos

$$
4 a \sqrt{(x+c)^{2}+y^{2}}=4 a^{2}+4 x c
$$




$$
\begin{aligned}
& \Rightarrow 4 a \sqrt{(x+c)^{2}+y^{2}}=4 a^{2}+x^{2}+4 x c+c^{2}-c^{2}+y^{2}-y^{2}-x^{2} \\
& \Rightarrow x^{2}-2 x c+c^{2}+y^{2}=4 a^{2}-4 a \sqrt{(x+c)^{2}+y^{2}}+(x+c)^{2}+y^{2} \\
& \Rightarrow x^{2}-2 x c+c^{2}+y^{2}=4 a^{2}-4 a \sqrt{(x+c)^{2}+y^{2}}+\left(\sqrt{(x+c)^{2}+y^{2}}\right)^{2} \\
& \Rightarrow(x-c)^{2}+y^{2}=\left(2 a-\sqrt{(x+c)^{2}+y^{2}}\right)^{2} .
\end{aligned}
$$

Novamente temos duas possibilidades:

$$
\begin{aligned}
& \sqrt{(x-c)^{2}+y^{2}}=2 a-\sqrt{(x+c)^{2}+y^{2}} \\
& \text { ou } \\
& -\sqrt{(x-c)^{2}+y^{2}}=2 a-\sqrt{(x+c)^{2}+y^{2}}
\end{aligned}
$$

Vamos trabalhar agora com a Eq. 2. Multiplicando a equação por 4, temos

$$
\begin{aligned}
& -4 a \sqrt{(x+c)^{2}+y^{2}}=4 a^{2}+4 x c \\
& \Rightarrow-4 a \sqrt{(x+c)^{2}+y^{2}}=4 a^{2}+x^{2}+4 x c+c^{2}-c^{2}+y^{2}-y^{2}-x^{2} \\
& \Rightarrow x^{2}-2 x c+c^{2}+y^{2}=4 a^{2}+4 a \sqrt{(x+c)^{2}+y^{2}}+(x+c)^{2}+y^{2} \\
& \Rightarrow x^{2}-2 x c+c^{2}+y^{2}=4 a^{2}+4 a \sqrt{(x+c)^{2}+y^{2}}+\left(\sqrt{(x+c)^{2}+y^{2}}\right)^{2} \\
& \Rightarrow(x-c)^{2}+y^{2}=\left(2 a+\sqrt{(x+c)^{2}+y^{2}}\right)^{2}
\end{aligned}
$$

Assim como no caso da Eq. 1, trabalhando com a Eq. 2 obtemos duas equação:

$$
\begin{aligned}
& \sqrt{(x-c)^{2}+y^{2}}=2 a+\sqrt{(x+c)^{2}+y^{2}} \\
& \text { ou } \\
& -\sqrt{(x-c)^{2}+y^{2}}=2 a+\sqrt{(x+c)^{2}+y^{2}}
\end{aligned}
$$

A expressão $\sqrt{(x-c)^{2}+y^{2}}$, ou ainda, $\sqrt{(x-c)^{2}+(y-0)^{2}}$ é a distância entre $P$ e $F_{1}$. E $\sqrt{(x+c)^{2}+y^{2}}=\sqrt{(x-(-c))^{2}+(y-0)^{2}}$ é a distância entre $\mathrm{P}$ e $F_{2}$. Podemos então apresentar as quatro equações Eq.1.1, Eq.1.2, Eq.2.1 e Eq.2.2, em termos de distâncias:

$$
\begin{array}{lc}
\operatorname{dist}\left(P, F_{1}\right)=2 a-\operatorname{dist}\left(P, F_{2}\right) & (\text { Eq.D.1.1), } \\
-\operatorname{dist}\left(P, F_{1}\right)=2 a-\operatorname{dist}\left(P, F_{2}\right) & (\text { Eq.D.1.2), } \\
\operatorname{dist}\left(P, F_{1}\right)=2 a+\operatorname{dist}\left(P, F_{2}\right) & (\text { Eq.D.2.1), } \\
-\operatorname{dist}\left(P, F_{1}\right)=2 a+\operatorname{dist}\left(P, F_{2}\right) & \text { (Eq.D.2.2). }
\end{array}
$$

A equação Eq.D.1.1 nos dá $\operatorname{dist}\left(P, F_{1}\right)+\operatorname{dist}\left(P, F_{2}\right)=2 a$, o que prova que $P$ está na elipse de focos $F_{1}$ e $F_{2}$ e constante $2 a$.

Para terminar a demostração vamos verificar que as outras três equações não são possíveis. 
A equação Eq.D.1.2 nos dá $\operatorname{dist}\left(P, F_{1}\right)+2 a=\operatorname{dist}\left(P, F_{2}\right)$. Pela desigualdade triangular

$$
\operatorname{dist}\left(F_{1}, F_{2}\right)+\operatorname{dist}\left(F_{1}, P\right) \geq \operatorname{dist}\left(F_{2}, P\right) .
$$

Substituindo $\operatorname{dist}\left(F_{1}, F_{2}\right)$ por $2 c$ na desigualdade triangular mencionada, temos

$$
2 c+\operatorname{dist}\left(F_{1}, P\right) \geq \operatorname{dist}\left(F_{2}, P\right) .
$$

Substituindo $\operatorname{dist}\left(F_{2}, P\right)$ por $\operatorname{dist}\left(P, F_{1}\right)+2 a$ de acordo com Eq.D.1.2, ficamos com

$$
2 c+\operatorname{dist}\left(F_{1}, P\right) \geq \operatorname{dist}\left(P, F_{1}\right)+2 a \text {. }
$$

Simplificando, temos $2 c \geq 2 a$. O que não pode ocorrer, pois $c=\sqrt{a^{2}-b^{2}}<a$.

Da Eq.D.2.1 temos $\operatorname{dist}\left(P, F_{1}\right)=2 a+\operatorname{dist}\left(P, F_{2}\right)$. Pela desigualdade triangular

$$
\operatorname{dist}\left(F_{2}, P\right)+\operatorname{dist}\left(F_{1}, F_{2}\right) \geq \operatorname{dist}\left(F_{1}, P\right) .
$$

Substituindo $\operatorname{dist}\left(F_{1}, F_{2}\right)$ por $2 c$ na desigualdade triangular mencionada, temos

$$
\operatorname{dist}\left(F_{2}, P\right)+2 c \geq \operatorname{dist}\left(F_{1}, P\right) .
$$

Substituindo $\operatorname{dist}\left(F_{1}, P\right)$ por $\operatorname{dist}\left(P, F_{2}\right)+2 a$ de acordo com Eq.D.2.1, ficamos com

$$
\operatorname{dist}\left(F_{2}, P\right)+2 c \geq \operatorname{dist}\left(P, F_{2}\right)+2 a .
$$

Simplificando, temos $2 c \geq 2 a$. O que não pode ocorrer, pois $c=\sqrt{a^{2}-b^{2}}<a$.

Por último, a Eq.D.2.2 nos diz que $-\operatorname{dist}\left(F_{1}, P\right)=2 a+\operatorname{dist}\left(F_{2}, P\right)$. No lado esquerdo da equação temos um número negativo, enquanto que no lado direito temos um número positivo, o que é um absurdo.

Provamos então que apenas a equação Eq.D.1.1 é compatível. Logo $P$ está na elipse de focos $F_{1}$ e $F_{2}$ e constante $2 a$. 
B

\section{Apêndice com Recíproca da Eq. da Hipérbole}

Seja $P=(x, y)$ um ponto do plano tal que $\frac{x^{2}}{a^{2}}-\frac{y^{2}}{b^{2}}=1$ para $a>0$ e $b>0$. Vamos provar que $P$ pertence à hipérbole de focos $F_{1}=(c, 0)$ e $F_{2}=(-c, 0)$ e constante $2 a$, onde $c=\sqrt{a^{2}+b^{2}}$, ou seja, vamos provar que $\left|\operatorname{dist}\left(P, F_{1}\right)-\operatorname{dist}\left(P, F_{2}\right)\right|=2 a$.

Vamos fazer algumas observações sobre os pontos que estamos considerando antes de começarmos as contas. Como $c=\sqrt{a^{2}+b^{2}}$ e $a$ e $b$ são positivos, temos $c>0$, e portanto $F_{1} \neq F_{2}$. Além disso, $F_{1} \neq P$ e $F_{2} \neq P$, pois as coordenadas de $F_{1}$ e de $F_{2}$ não satisfazem a equação $\frac{x^{2}}{a^{2}}-\frac{y^{2}}{b^{2}}=1$. De fato, colocando as coordenadas de $F_{1}$ ou de $F_{2}$ na equação, teríamos $\frac{c^{2}}{a^{2}}-\frac{0^{2}}{b^{2}}=1$, ou seja, $c^{2}=a^{2}$, o que não pode acontecer uma vez que $c^{2}=a^{2}+b^{2}$ e $b>0$.

Substituindo $-b^{2}$ por $a^{2}-c^{2}$ na equação $\frac{x^{2}}{a^{2}}-\frac{y^{2}}{b^{2}}=1$, ficamos com:

$$
\frac{x^{2}}{a^{2}}+\frac{y^{2}}{a^{2}-c^{2}}=1 \quad \text { (Eq.B) }
$$

Observamos que a Eq.B é idêntica à Eq.A do Apêndice anterior que trata o caso da elipse. A diferença está apenas na relação entre as constantes $a$ e $c$, portanto com as mesmas manipulações algébricas chegamos nas quatro equações:

$$
\begin{aligned}
& \sqrt{(x-c)^{2}+y^{2}}=2 a-\sqrt{(x+c)^{2}+y^{2}} \quad \text { (Eq.1.1), } \\
& -\sqrt{(x-c)^{2}+y^{2}}=2 a-\sqrt{(x+c)^{2}+y^{2}} \\
& \sqrt{(x-c)^{2}+y^{2}}=2 a+\sqrt{(x+c)^{2}+y^{2}}
\end{aligned}
$$

Em termos de distâncias:

$$
\begin{array}{lc}
\operatorname{dist}\left(P, F_{1}\right)=2 a-\operatorname{dist}\left(P, F_{2}\right) & (\text { Eq.D.1.1), } \\
-\operatorname{dist}\left(P, F_{1}\right)=2 a-\operatorname{dist}\left(P, F_{2}\right) & (\text { Eq.D.1.2), } \\
\operatorname{dist}\left(P, F_{1}\right)=2 a+\operatorname{dist}\left(P, F_{2}\right) & (\text { Eq.D.2.1), } \\
-\operatorname{dist}\left(P, F_{1}\right)=2 a+\operatorname{dist}\left(P, F_{2}\right) & \text { (Eq.D.2.2). }
\end{array}
$$

A equação Eq.D.1.2 é equivalente à equação $\operatorname{dist}\left(P, F_{2}\right)-\operatorname{dist}\left(P, F_{1}\right)=$ $2 a$. E Eq.D.2.1 é equivalente à equação $\operatorname{dist}\left(P, F_{1}\right)-\operatorname{dist}\left(P, F_{2}\right)=2 a$. Dizer 
que $P$ satisfaz $\operatorname{dist}\left(P, F_{2}\right)-\operatorname{dist}\left(P, F_{1}\right)=2 a$ ou $\operatorname{dist}\left(P, F_{1}\right)-\operatorname{dist}\left(P, F_{2}\right)=2 a$ é equivalente a dizer que $\mathrm{P}$ satisfaz $\left|\operatorname{dist}\left(P, F_{1}\right)-\operatorname{dist}\left(P, F_{2}\right)\right|=2 a$ e portanto $P$ pertence à hipérbole de focos $F_{1}$ e $F_{2}$ e constante $2 a$.

Para terminar a demostração basta verificar que as equações Eq.D.1.1 e Eq.D.2.2 não são possíveis.

Da Eq.D.1.1 temos $\operatorname{dist}\left(P, F_{1}\right)+\operatorname{dist}\left(P, F_{2}\right)=2 a$. Pela desigualdade triangular

$$
\operatorname{dist}\left(F_{2}, P\right)+\operatorname{dist}\left(F_{1}, P\right) \geq \operatorname{dist}\left(F_{1}, F_{2}\right) .
$$

Substituindo dist $\left(F_{1}, F_{2}\right)$ por $2 c$ na desigualdade triangular mencionada, temos

$$
\operatorname{dist}\left(F_{2}, P\right)+\operatorname{dist}\left(F_{1}, P\right) \geq 2 c .
$$

$\operatorname{Substituindo} \operatorname{dist}\left(P, F_{1}\right)+\operatorname{dist}\left(P, F_{2}\right)$ por $2 a$ de acordo com Eq.D.1.1, ficamos com $2 a \geq 2 c$. O que não pode ocorrer, pois $c=\sqrt{a^{2}+b^{2}}>a$.

Por último, a Eq.D.2.2 nos $\operatorname{diz}$ que $-\operatorname{dist}\left(F_{1}, P\right)=2 a+\operatorname{dist}\left(F_{2}, P\right)$. No lado esquerdo da equação temos um número negativo, enquanto que no lado direito temos um número positivo, o que é um absurdo.

Logo $P$ está na hipérbole de focos $F_{1}$ e $F_{2}$ e constante $2 a$. 